\title{
Substrate Effect on the Optical Reflectance and Transmittance of Thin-Film Structures
}

\author{
Anatoly Barybin and Victor Shapovalov \\ Electronics Department, Saint Petersburg State Electrotechnical University, Saint Petersburg 197376, Russia \\ Correspondence should be addressed to Anatoly Barybin, barybin@mail.ru
}

Received 8 September 2010; Accepted 3 November 2010

Academic Editor: Robert G. Elliman

Copyright ( $) 2010$ A. Barybin and V. Shapovalov. This is an open access article distributed under the Creative Commons Attribution License, which permits unrestricted use, distribution, and reproduction in any medium, provided the original work is properly cited.

\begin{abstract}
A rigorous and consistent approach is demonstrated to develop a model of the $4 \mathrm{M}$ structure (the four-media structure of a film on a substrate of finite thickness). The general equations obtained for the reflectance and transmittance spectra of the $4 \mathrm{M}$ structure are simplified by employing a procedure of the so-called device averaging to reduce them to a succinct form convenient for processing of experimental spectra for the structures with a thick substrate. The newly derived equations are applied to two special cases: (i) an arbitrary film on highly absorbing substrates and (ii) a slightly absorbing film on transparent substrates. The reflectance and transmittance spectra represented in the simplified (with the device averaging) form have a practical application for determining the film thickness and optical constants from experimental spectra by using the known techniques.
\end{abstract}

\section{Introduction}

Nowadays, there are diverse modern optical measurement methods, which are based on electromagnetic theory applied to interference and absorption phenomena in thin-film layered structures. Advance in developing new physical principles of optical measurements has been achieved by extensive works and intensive efforts of a great number of scientists and researchers. Theoretical and practical knowledge in thinfilm optics and optical measurements has been accumulated in numerous scientific publications; well-known books and reviews may be quoted as an example [1-12]. They describe optical properties of various films, techniques of measuring the reflectance and transmittance spectra, and calculations of the film thickness and optical constants from experimental spectra.

The present interest in these subjects is caused by the wide application of thin-film structures in various optical devices and the fact that the optical methods of measurement give necessary information about the structural and physical properties of films that are used in optics and microelectronics [13]. Optical properties of thin films present a vital issue due to the increase of thin films quality standards in traditional areas of application [14] and the possibility to solve some recently discovered technical tasks only in thinfilm performance; first, we refer to biomedical and ecological applications [15-19].

The problem of determining the film thickness and dispersion properties of optical constants is usually solved by analyzing experimental spectra of the reflection and/or transmission for film-on-substrate structures of interest. Results of the analysis depend on a choice of physical model connecting experimentally measured spectra with geometrical and optical parameters of the structure under study. In order to obtain some reliable relations for practical calculations, as applied to processing of the results of measurements, it is necessary to develop a physically correct model of the thin-film structure, allowing interference and absorption in both the film and substrate materials.

Various physical approaches and mathematical models describing the optical spectra for thin-film structures can be found in the literature. They usually present identical results in complicated forms difficult for comparison [112, 20-27]. A wide variety of different formulae available in the literature, which have been also derived by using dissimilar designations, essentially complicate the choice of the application to a given practical problem. In search of reliable technique for calculations needed for processing the 


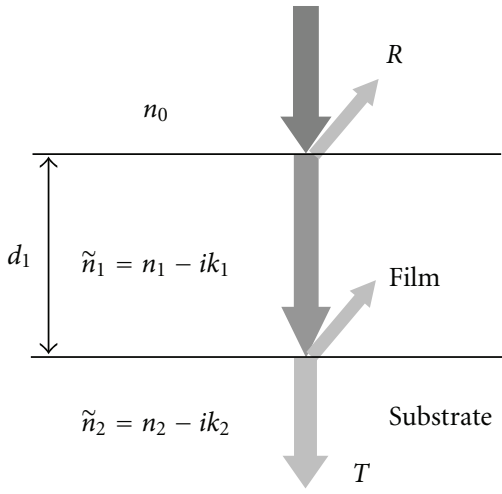

(a)

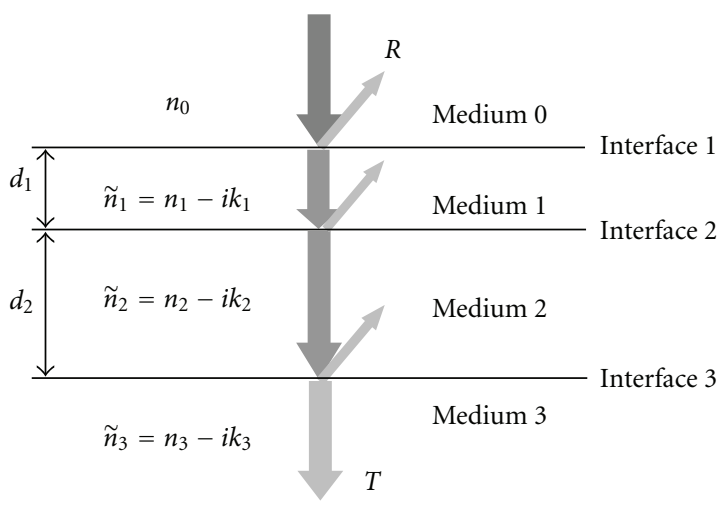

(b)

Figure 1: Schematic geometry for: (a) the $3 \mathrm{M}$ structure with a semiinfinite substrate and (b) the $4 \mathrm{M}$ structure with a single film (medium 1 ) on a substrate of finite thickness $d_{2}$ (medium 2); media 0 and 3 correspond to the input and output ones.

results of spectral measurements an experimenter has to waste time on mathematical transformations to compare the theoretical expressions obtained by different authors.

Although most theoretical approaches generally begin with studying a system of multiple layers $[1,5,6,8,9]$, practical application is found only for the simplest threemedia structure (3M structure) appropriate to a single film on a semiinfinite substrate. In particular, Heavens [10] analyzes some special cases of the $3 \mathrm{M}$ structure, as applied to the measurement of optical constants of thin films. The same author has developed the more general model for the fourmedia structure (4M structure) appropriate to a single film on a substrate of finite thickness in the earlier published book [5]. This model looks more practical in the application to optical measurements, as the expressions for the reflectance and transmittance of the $4 \mathrm{M}$ structure in addition to the finite substrate thickness contain also optical parameters of both the input and output media adjacent to the film and substrate. However, the general formulae obtained by Heavens [5] describing the $4 \mathrm{M}$ structure turn out to be extremely complicated, which restricts their practical applicability to the processing of experimental spectra.

Section 2 is devoted to a critical analysis of existing models for the reflectance and transmittance spectra and formulates the theoretical problem for further investigation. Section 3 describes the matrix approach to evaluation of the reflectance and transmittance of multilayer structures. The general matrix relations derived by involving the Fresnel reflection coefficients of multiple interfaces are employed to the special cases of the $3 \mathrm{M}$ and $4 \mathrm{M}$ structures. In Section 4 formulae for the reflectance and transmittance originally expressed in terms of the Fresnel coefficients are adduced in a physical form containing the optical constants of media (refractive and absorptive indices) together with the geometrical parameters of the considered structures. Mathematical details transforming the relevant formulae to the physical form are set forth in Appendix A. The general formulae are simplified by using a procedure of the socalled device averaging (see Appendix B) to reduce them to a succinct form convenient for the processing of experimental spectra for structures with a thick substrate. In Section 5, the simplified equations are applied to two special cases: (i) an arbitrary film on highly absorbing substrates and (ii) a slightly absorbing film on transparent substrates.

\section{Formulation of the Problem}

We shall restrict our consideration to normal incidence of light from a transparent input medium of the real refractive index $n_{0}$. In the general case, the film under examination (numbered by 1 ) has optical losses so that its refractive index is complex and dispersive: $\tilde{n}_{1}(\lambda)=n_{1}(\lambda)-i k_{1}(\lambda)$ with the real refractive and absorptive indices $n_{1}(\lambda)$ and $k_{1}(\lambda)$ being functions of the light wavelength $\lambda$. Depending on application of the thin-film structure, its substrate (numbered by 2) is produced from different materials including transparent dielectrics as well as absorbing metals or semiconductors. Thus, in general, the refractive index of substrate also has the complex form $\tilde{n}_{2}(\lambda)=n_{2}(\lambda)-i k_{2}(\lambda)$.

Examination of various experimental situations allows one to select the two above-mentioned thin-film structures, which are available for thorough mathematical study. They are depicted in Figure 1: (a) the three-media structure (3M structure) appropriate to a film of thickness $d_{1}$ on a semiinfinite substrate and (b) the four-media structure (4M structure) appropriate to a single film of thickness $d_{1}$ on a substrate of finite thickness $d_{2}$. Media 0 and 3 may be considered respectively an input medium (non absorbing one with real index $n_{0}$ ) and an output medium (absorbing one with complex index $\tilde{n}_{3}(\lambda)=n_{3}(\lambda)-i k_{3}(\lambda)$ ). The reflected flows of light are shown in Figure 1 at some angle only for pictorial rendition; the case under consideration has the normal incidence.

The main task of theoretical analysis is a derivation of mathematical expressions for the spectra of reflectance $R(\lambda)$ and transmittance $T(\lambda)$, which can be applied to models of the $3 \mathrm{M}$ and $4 \mathrm{M}$ structures. In our opinion, the final expressions for $R(\lambda)$ and $T(\lambda)$ within the framework of any mathematical model should, first, correctly describe physical phenomena occurring under action of light (wave 
interference and energy absorption) and, second, have a sufficiently succinct form to be convenient for both the physical interpretation and numerical computation. From succinctness standpoint, an expression for the transmittance spectrum given by Swanepoel $[24,25]$ is more successful, unlike many others of cumbersome forms $[5,9,10,12]$. However, Swanepoel's approach casts some doubts upon its mathematical justification, as shown below.

In Swanepoel's notation [24], the transmittance spectrum of a slightly absorbing film $\left(k_{1}^{2} \ll n_{1}^{2}\right)$ on a transparent substrate $\left(k_{2}=0\right)$ has the following form:

$$
T(\lambda)=\frac{A x}{B-C x \cos \varphi+D x^{2}},
$$

where $x=\exp \left(-\alpha d_{1}\right), \alpha=4 \pi k_{1} / \lambda, \varphi=4 \pi n_{1} d_{1} / \lambda$, while $A, B, C$, and $D$ are the coefficients depending on optical constants of media taken into account by the model under consideration. In publications, there are different expressions for these coefficients; in particular, authors in [21, 23] apply them in the following form (with notation of $n_{m}, m=0,1,2$, according to Figure 1(a))

$$
\begin{gathered}
A=16 n_{0} n_{1}^{2} n_{2}, \quad C=-2\left(n_{0}^{2}-n_{1}^{2}\right)\left(n_{1}^{2}-n_{2}^{2}\right), \\
B=\left(n_{0}+n_{1}\right)^{2}\left(n_{1}+n_{2}\right)^{2}, \quad D=\left(n_{0}-n_{1}\right)^{2}\left(n_{1}-n_{2}\right)^{2} .
\end{gathered}
$$

When using (1)-(2), one implicitly means that a substrate is semiinfinite, which conforms to the $3 \mathrm{M}$ structure (Figure 1(a)), and then a receiver of transmitted light should be placed inside the substrate. In fact, such is not the case and the light receiver must be outside a substrate. So, the model of semiinfinite substrate does not work in real situations. Consequently, (1)-(2) yield some higher values of the transmittance because they disregard an additional reflection from another interface of a real substrate (for a glass one the error is around 4\% [4]). For this reason, in order to take into account the above-stated fact, Swanepoel $[24,25]$, followed by other authors [26-31], has applied (1) for the transmittance of a single film on a transparent substrate and suggested that the coefficients $A$ and $C$ should be left in the unchanged form (2), while $B$ and $D$ are written as follows:

$$
\begin{gathered}
A=16 n_{0} n_{1}^{2} n_{2}, \quad C=-2\left(n_{0}^{2}-n_{1}^{2}\right)\left(n_{1}^{2}-n_{2}^{2}\right), \\
B=\left(n_{0}+n_{1}\right)^{3}\left(n_{1}+n_{2}^{2}\right), \quad D=-\left(n_{0}-n_{1}\right)^{3}\left(n_{1}-n_{2}^{2}\right) .
\end{gathered}
$$

A distinguishing feature of expressions (3) as compared with (2) consists of appearance of the third power of the first parentheses and asymmetry in powers of items inside the second parentheses for the coefficients $B$ and $D$. Both facts are very doubtful but have no mathematical substantiation by Swanepoel [24]. In effect, (1) and (3), as applied to the 3M structure in [24], implicitly correspond to the $4 \mathrm{M}$ structure shown in Figure 1(b). However, these formulae do not take into account the optical constants of an output medium (numbered by 3), quite apart from lack of the finite substrate thickness $d_{2}$ inherent in the $4 \mathrm{M}$ structure. The above-stated reasons cause some doubts on the correctness of Swanepoel's approach [24, 25].

Consequently, the problem of reflection and transmission of light, as applied to the film-on-substrate structures especially with regard for optical absorption, can correctly be solved only within the scope of the $4 \mathrm{M}$ structure model. The $3 \mathrm{M}$ structure model is of practical interest solely in the special case that the substrate has high optical losses, which is typical for semiconductor and metal substrates. In this case, the sole reflectance spectrum $R(\lambda)$ is practically accessible from experimental measurements, unlike the transmittance spectrum $T(\lambda)$. As proved later, both the spectra $R(\lambda)$ and $T(\lambda)$ for the $3 \mathrm{M}$ structures can be theoretically obtained as a special case of the general physical situation examined within the framework of the $4 \mathrm{M}$ structure model.

Certain expressions for the spectra $R(\lambda)$ and $T(\lambda)$ of the $4 \mathrm{M}$ structure were also derived by Heavens and represented in the following form [5]:

$$
R(\lambda)=\frac{t_{13}^{2}+u_{13}^{2}}{p_{13}^{2}+q_{13}^{2}}, \quad T(\lambda)=\frac{l_{13}^{2}+m_{13}^{2}}{p_{13}^{2}+q_{13}^{2}} .
$$

Here, the items $l_{13}^{2}, m_{13}^{2}, p_{13}^{2}, q_{13}^{2}, t_{13}^{2}$, and $u_{13}^{2}$ depend on the optical constants $\tilde{n}_{m}=n_{m}-i k_{m}$ of media $(m=1,2,3)$, their thicknesses $d_{m}$, and the light wavelength $\lambda$.

Expressions (4) only look succinct because the items $l_{13}^{2}$, $m_{13}^{2}$, and so forth are very involved and physically tangled, being composed of a number of other terms by successive substitution of them one after the other. Such a "stepby-step" construction of Heavens' items, unlike the simple one of Swanepoel's coefficients (3), practically eliminates the processing ability of formulae (4), as applied to the experimental spectra $R(\lambda)$ and $T(\lambda)$. Also, this makes it difficult, if not impossible, to provide insight into influence of physical parameters of the structure on its optical spectra. We do not adduce here expressions for $l_{13}^{2}, m_{13}^{2}$, and so forth, because of their complexity and refer an interested reader to the original book [5] or paper [32], which have also given them.

Formulae (4) describe the physical situation corresponding to interference of waves transmitted and reflected by three interfaces, as shown in Figure 1(b). These formulae are applicable to any film-on-substrate structure with arbitrary properties including a double absorbing film (media 1 and 2) on semiinfinite absorbing substrates (medium 3 ).

Figure 2 shows spectra $R(\lambda)$ and $T(\lambda)$ of film $\mathrm{Ta}_{2} \mathrm{O}_{5}$ on a quartz-glass substrate $\left(n_{0}=n_{3}=1, n_{1}=2.21\right.$, $n_{2}=1.472, k_{1}=k_{2}=k_{3}=0, d_{1}=1 \mu \mathrm{m}$, and $d_{2}=$ $500 \mu \mathrm{m})$ calculated on the basis of Heavens' formulae (4). In both spectra, there are oscillations of two types caused by wave interference in the thin film $\mathrm{Ta}_{2} \mathrm{O}_{5}$ (slow oscillations) and in the thick substrate (fast oscillations in the form of a "beard"). Space periods of these oscillations differ from each other by the factor $\eta \equiv n_{2} d_{2} / n_{1} d_{1}$, which exceeds two orders in magnitude for the given instance. However, when measuring the spectra an experimental result takes the form of a dashed curve in Figure 2 caused by interference only in the thin film $\mathrm{Ta}_{2} \mathrm{O}_{5}$. This stems from the fact that 


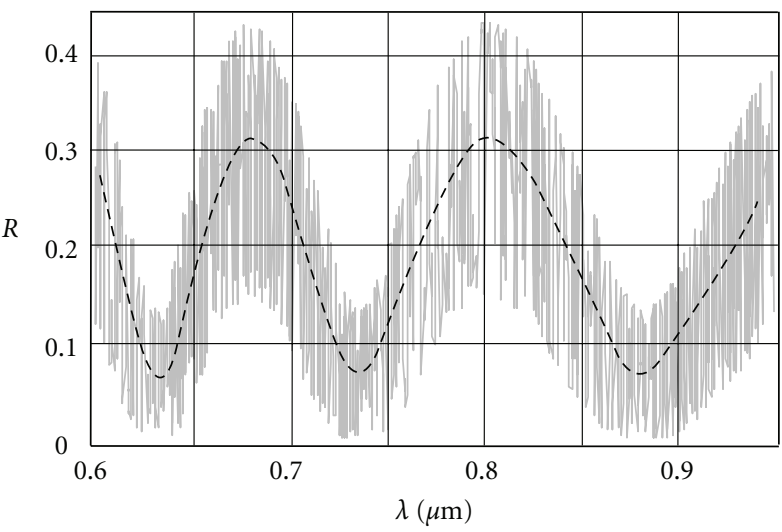

(a)

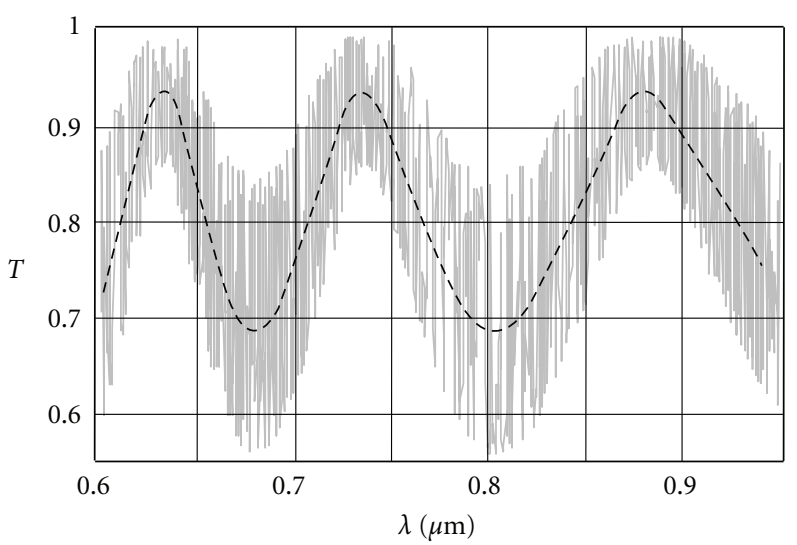

(b)

Figure 2: Reflectance $(R)$ and transmittance $(T)$ spectra of the $\mathrm{Ta}_{2} \mathrm{O}_{5}$-quartz-glass structure calculated from (4) and shown by gray lines in the form of a "beard". Dashed curves on the spectra result from measurements by using a spectrophotometer with operating slit of finite width.

the operating slit of a spectrophotometer is not infinitely narrow but has finite width (of a few nanometers). Because of this, the input irradiation is not monochromatic, and the fast oscillations caused by interference in the thick substrate with $n_{2} d_{2} \gg n_{1} d_{1}$ prove to be averaged down to zero, as is mathematically justified in Appendix B. In other words, any spectrophotometer with an operating slit of finite width performs a procedure that will be called the device averaging. As a result of this procedure, the "beard" produced by interference oscillations in a thick substrate is liquidated, and only the thin-film interference curve similar to dashed ones in Figure 2 is left in experimental spectra.

Our subsequent task consists of deriving the analytical expressions for $R(\lambda)$ and $T(\lambda)$ in such a form that allows us to carry out the device averaging.

\section{General Matrix Theory for Multilayer Structures}

3.1. Desired Form of the Fresnel Reflection Coefficients. Consider any two adjacent layers of the multilayer structure under consideration, say, the $(m-1)$ th and $m$ th absorbing layers specified by the complex refractive indices

$$
\tilde{n}_{m-1}=n_{m-1}-i k_{m-1}, \quad \tilde{n}_{m}=n_{m}-i k_{m},
$$

where $m=1,2, \ldots$ and $\tilde{n}_{0}=n_{0}$ since $k_{0} \equiv 0$ for a nonabsorbing input medium. Each $m$ th interface is characterized by the Fresnel reflection and transmission coefficients $r_{m}$ and $t_{m}=1+r_{m}$ defined as [1-7]

$$
r_{m}=\frac{\tilde{n}_{m-1}-\tilde{n}_{m}}{\tilde{n}_{m-1}+\tilde{n}_{m}}, \quad t_{m}=\frac{2 \tilde{n}_{m-1}}{\tilde{n}_{m-1}+\tilde{n}_{m}}
$$

Heavens [5] represents the Fresnel reflection coefficient $r_{m}$ as a sum of its real and imaginary parts

$$
r_{m}=g_{m}+i h_{m}
$$

where in accordance with (5) and (6), we have

$$
\begin{aligned}
& g_{m}=\frac{\left|\tilde{n}_{m-1}\right|^{2}-\left|\tilde{n}_{m}\right|^{2}}{\left(n_{m-1}+n_{m}\right)^{2}+\left(k_{m-1}+k_{m}\right)^{2}}, \\
& h_{m}=\frac{2\left(n_{m-1} k_{m}-n_{m} k_{m-1}\right)}{\left(n_{m-1}+n_{m}\right)^{2}+\left(k_{m-1}+k_{m}\right)^{2}},
\end{aligned}
$$

with the squared moduli of the refractive indices (5) being equal to

$$
\left|\tilde{n}_{m-1}\right|^{2}=n_{m-1}^{2}+k_{m-1}^{2}, \quad\left|\tilde{n}_{m}\right|^{2}=n_{m}^{2}+k_{m}^{2} .
$$

Unlike Heavens' approach, for our analysis, it is more convenient to apply for $r_{m}$ instead of (7) the exponential form

$$
r_{m}=\rho_{m} \exp \left(i \theta_{m}\right)
$$

The squared modulus $\rho_{m}^{2}$ and phase $\theta_{m}$ of the Fresnel reflection coefficient (7) or (10) are defined by the following relations

$$
\begin{gathered}
\rho_{m}^{2}=g_{m}^{2}+h_{m}^{2}=\frac{\left|\tilde{n}_{m-1}-\tilde{n}_{m}\right|^{2}}{\left|\tilde{n}_{m-1}+\tilde{n}_{m}\right|^{2}} \equiv \frac{\mathcal{N}_{-m}}{\mathcal{N}_{+m}}, \\
\tan \theta_{m}=\frac{h_{m}}{g_{m}}=\frac{2\left(n_{m-1} k_{m}-n_{m} k_{m-1}\right)}{\left|\tilde{n}_{m-1}\right|^{2}-\left|\tilde{n}_{m}\right|^{2}},
\end{gathered}
$$

where we have introduced the following quantities

$$
\begin{aligned}
\mathcal{N}_{ \pm m} & \equiv\left|\tilde{n}_{m-1} \pm \tilde{n}_{m}\right|^{2}=\left(n_{m-1} \pm n_{m}\right)^{2}+\left(k_{m-1} \pm k_{m}\right)^{2} \\
& =\left(\left|\tilde{n}_{m-1}\right|^{2}+\left|\tilde{n}_{m}\right|^{2}\right) \pm 2\left(n_{m-1} n_{m}+k_{m-1} k_{m}\right) .
\end{aligned}
$$

In the absence of optical absorption $\left(k_{m-1}=k_{m}=0\right)$, expression (6) for $r_{m}$ gives purely real values (negative or positive) so that $r_{m}<0$ or $r_{m}>0$ when, respectively, $n_{m-1}<n_{m}$ or $n_{m-1}>n_{m}$. Yet, the exponential form (10) 
does not display explicitly this property. Indeed, from (12), it follows that in the lossless situation $\tan \theta_{m}=0$ and by definition always $\rho_{m}>0$. In order for the above-stated property to be explicitly displayed from relation (10), it is necessary to require the phase $\theta_{m}$ to be positively defined. This requirement is readily realized by considering the phase angle $\theta_{m}$ to be a function of the quantity

$$
\begin{aligned}
x_{m} & =s_{m} \frac{h_{m}}{g_{m}} \equiv \frac{h_{m}}{\left|g_{m}\right|} \\
& =\frac{2\left(n_{m-1} k_{m}-n_{m} k_{m-1}\right)}{\left.|| \widetilde{n}_{m-1}\right|^{2}-\left|\widetilde{n}_{m}\right|^{2} \mid} \\
& =\frac{2\left(n_{m-1} k_{m}-n_{m} k_{m-1}\right)}{\left|\left(n_{m-1}^{2}-n_{m}^{2}\right)+\left(k_{m-1}^{2}-k_{m}^{2}\right)\right|},
\end{aligned}
$$

where the sign of $g_{m}$ is inserted as

$$
\begin{aligned}
s_{m} & =\frac{g_{m}}{\left|g_{m}\right|} \\
& =\operatorname{sgn}\left\{\left|\tilde{n}_{m-1}\right|^{2}-\left|\tilde{n}_{m}\right|^{2}\right\} \\
& = \begin{cases}-1 & \text { if }\left|\tilde{n}_{m-1}\right|<\left|\tilde{n}_{m}\right|, \\
+1 & \text { if }\left|\tilde{n}_{m-1}\right|>\left|\tilde{n}_{m}\right| .\end{cases}
\end{aligned}
$$

From formulae (12) and (14) explained by Figure 3 it follows that

$$
\begin{aligned}
& \theta_{m}=\arctan \left(s_{m} x_{m}\right) \\
& = \begin{cases}\arctan \left(+x_{m}\right)=0+\phi_{m} & \text { for } s_{m}=+1 \\
\arctan \left(-x_{m}\right)=\pi-\phi_{m} & \text { for } s_{m}=-1,\end{cases} \\
& \exp \left(i \theta_{m}\right)=s_{m} \exp \left(i s_{m} \phi_{m}\right),
\end{aligned}
$$

where the new phase angle $\phi_{m}$ is defined by the following relation:

$$
\tan \phi_{m}=x_{m} \equiv \frac{h_{m}}{\left|g_{m}\right|}=\frac{2\left(n_{m-1} k_{m}-n_{m} k_{m-1}\right)}{\left.|| \tilde{n}_{m-1}\right|^{2}-\left|\tilde{n}_{m}\right|^{2} \mid} .
$$

Substitution of relation (17) into (10) yields the desired exponential form of the Fresnel reflection coefficient

$$
r_{m}=\bar{\rho}_{m} \exp \left(i \bar{\phi}_{m}\right) \quad \text { with } \bar{\rho}_{m}=s_{m} \rho_{m}, \bar{\phi}_{m}=s_{m} \phi_{m} .
$$

From (18) and (19), it evidently follows that in the lossless situation, when $\phi_{m}=0$, the Fresnel coefficient $r_{m}=$ $s_{m} \rho_{m}<0$ or $>0$ depending on whether $n_{m-1}<n_{m}$ or $n_{m-1}>n_{m}$, which is what we set out to obtain.

3.2. Matrix Approach to Evaluating the Reflectance and Transmittance. Let us consider the $(m-1)$ th and $m$ th layers which are incorporated into the $\mathrm{N}$-media (including input and output ones) structure (NM structure) and depicted in Figure 4. Electromagnetic fields in each of them are formed by superposition of two plane waves-positive going (of a complex amplitude marked by superscript + ) and negative

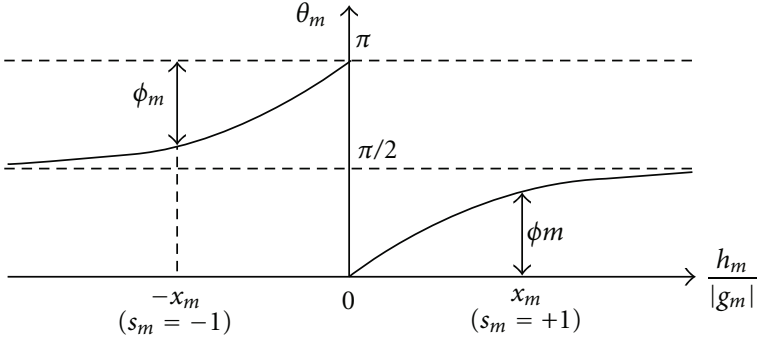

Figure 3: Illustration of the phase angles $\theta_{m}$ and $\phi_{m}$ for representing the Fresnel reflection coefficient in the exponential form $r_{m}=$ $\rho_{m} \exp \left(i \theta_{m}\right)=s_{m} \rho_{m} \exp \left(i s_{m} \phi_{m}\right)$.

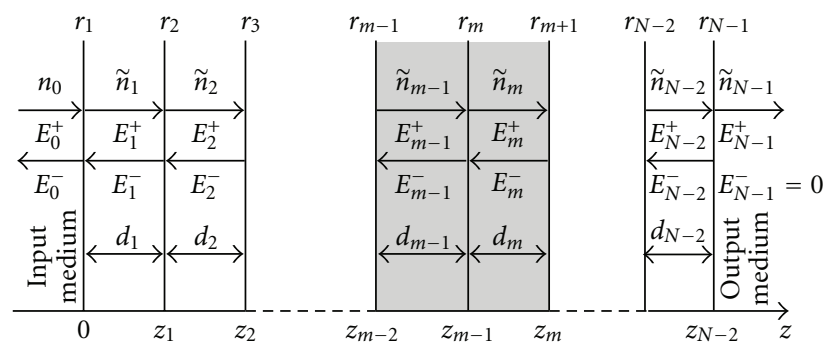

FIGURE 4: Schematic geometry of the multilayer structure consisting of $N$ media (including the input/output ones) with two highlighted $(m-1)$ th and $m$ th layers having an interface at $z_{m-1}$ specified by the Fresnel reflection coefficient $r_{m}=s_{m} \rho_{m} \exp \left(i s_{m} \phi_{m}\right)$.

going (of a complex amplitude marked by superscript -) with respect to the axis $z$ directed transversely to interfaces. As seen from Figure 4, light travels from an input medium to output one. So, for the $m$ th layer the positive-going wave of amplitude $E_{m}^{+}$corresponds to light transmitted across its left boundary at $z_{m-1}$, while the negative-going wave of amplitude $E_{m}^{-}$- to light reflected from its right boundary at $z_{m}$.

We shall describe light propagation in the $m$ th absorbing medium of the complex refractive index $\tilde{n}_{m}=n_{m}-i k_{m}$ by using the wave factor $\exp \left(i \omega t \mp \gamma_{m} z\right)$, where the propagation constant

$$
\gamma_{m}=i \frac{2 \pi}{\lambda} \tilde{n}_{m} \equiv \alpha_{m}+i \beta_{m}
$$

consists of the amplitude and phase constants defined as

$$
\alpha_{m}=\frac{2 \pi}{\lambda} k_{m}, \quad \beta_{m}=\frac{2 \pi}{\lambda} n_{m} .
$$

Let us write down the electromagnetic fields inside the two layers under examination

(i) for the $(m-1)$ th layer located at $z_{m-2}<z<z_{m-1}$

$$
\begin{gathered}
E_{m-1}(z)=E_{m-1}^{+} \mathrm{e}^{-\gamma_{m-1}\left(z-z_{m-2}\right)}+E_{m-1}^{-} \mathrm{e}^{\gamma_{m-1}\left(z-z_{m-2}\right)}, \\
H_{m-1}(z)=\tilde{n}_{m-1} E_{m-1}^{+} \mathrm{e}^{-\gamma_{m-1}\left(z-z_{m-2}\right)}-\widetilde{n}_{m-1} E_{m-1}^{-} \mathrm{e}^{\gamma_{m-1}\left(z-z_{m-2}\right)},
\end{gathered}
$$


(ii) for the $m$ th layer located at $z_{m-1}<z<z_{m}$

$$
\begin{gathered}
E_{m}(z)=E_{m}^{+} \mathrm{e}^{-\gamma_{m}\left(z-z_{m-1}\right)}+E_{m}^{-} \mathrm{e}^{\gamma_{m}\left(z-z_{m-1}\right)}, \\
H_{m}(z)=\tilde{n}_{m} E_{m}^{+} \mathrm{e}^{-\gamma_{m}\left(z-z_{m-1}\right)}-\tilde{n}_{m} E_{m}^{-} \mathrm{e}^{\gamma_{m}\left(z-z_{m-1}\right)} .
\end{gathered}
$$

Imposing on the electromagnetic fields (22) and (23) at interface point $z=z_{m-1}$ the following matching conditions:

$$
E_{m-1}\left(z_{m-1}\right)=E_{m}\left(z_{m-1}\right), \quad H_{m-1}\left(z_{m-1}\right)=H_{m}\left(z_{m-1}\right),
$$

we couple the complex amplitudes in the $(m-1)$ th and $m$ th layers by the matrix relation

$$
\left(\begin{array}{c}
E_{m-1}^{+} \\
E_{m-1}^{-}
\end{array}\right)=\frac{1}{t_{m}} C_{m}\left(\begin{array}{c}
E_{m}^{+} \\
E_{m}^{-}
\end{array}\right)
$$

where $t_{m}$ is the Fresnel transmission coefficient (6) for the $m$ th interface at $z=z_{m-1}$. The coupling matrix appearing in (25)

$$
C_{m}=\left(\begin{array}{cc}
\mathrm{e}^{\Delta_{m-1}} & r_{m} \mathrm{e}^{\Delta_{m-1}} \\
r_{m} \mathrm{e}^{-\Delta_{m-1}} & \mathrm{e}^{-\Delta_{m-1}}
\end{array}\right) \text { with } \Delta_{m-1}=\gamma_{m-1} d_{m-1}
$$

is identical in form to those obtained previously $[5,9]$.

To express the input electric fields $E_{0}^{ \pm}$in terms of the output field $E_{N-1}^{+}$, it is necessary to successively multiply the coupling matrices (26) for all layers entering into the NMstructure depicted in Figure 4. As a result, we obtain from (25) the following relation:

$$
\left(\begin{array}{c}
E_{0}^{+} \\
E_{0}^{-}
\end{array}\right)=\frac{1}{t_{1} t_{2} \cdots t_{N-1}}\left(\begin{array}{cc}
a_{N} & b_{N} \\
c_{N} & d_{N}
\end{array}\right)\left(\begin{array}{c}
E_{N-1}^{+} \\
0
\end{array}\right)
$$

where $E_{N-1}^{-}=0$ for a semiinfinite output medium and the resultant coupling matrix is

$$
\left(\begin{array}{cc}
a_{N} & b_{N} \\
c_{N} & d_{N}
\end{array}\right)=C_{1} C_{2} \cdots C_{N-1}
$$

The reflectance and transmittance (defined as ratios of the reflected and transmitted power to the incident power) immediately follow from (27) as

$$
\begin{gathered}
R \equiv \frac{\left|E_{0}^{-}\right|^{2}}{\left|E_{0}^{+}\right|^{2}}=\frac{\left|c_{N}\right|^{2}}{\left|a_{N}\right|^{2}} \\
T \equiv \frac{n_{N-1}}{n_{0}} \frac{\left|E_{N-1}^{+}\right|^{2}}{\left|E_{0}^{+}\right|^{2}}=\frac{n_{N-1}}{n_{0}} \frac{\left|t_{1}\right|^{2}\left|t_{2}\right|^{2} \cdots\left|t_{N-1}\right|^{2}}{\left|a_{N}\right|^{2}} .
\end{gathered}
$$

Therefore, in order to obtain $R$ and $T$, it is necessary to find only two matrix elements for calculating $\left|a_{N}\right|^{2}$ and $\left|c_{N}\right|^{2}$ and also to know the Fresnel transmission coefficients $t_{m}$ of all interfaces $(m=1,2, \ldots, N-1)$ for which from (19) it follows that

$$
\left|t_{m}\right|^{2}=\left|1+r_{m}\right|^{2}=1+\bar{\rho}_{m}^{2}+2 \bar{\rho}_{m} \cos \bar{\phi}_{m} .
$$

Let us apply (26)-(29) to the special cases appropriate to the $3 \mathrm{M}$ structure (Figure $1(\mathrm{a})$ ) and the $4 \mathrm{M}$ structure (Figure 1(b)).
3.3. Reflectance and Transmittance of the $3 M$ Structure. For the $3 \mathrm{M}$ structure shown in Figure 1(a) the coupling matrix product equals

$$
\begin{aligned}
C_{1} C_{2} & =\left(\begin{array}{cc}
1 & r_{1} \\
r_{1} & 1
\end{array}\right)\left(\begin{array}{cc}
\mathrm{e}^{\Delta_{1}} & r_{2} \mathrm{e}^{\Delta_{1}} \\
r_{2} \mathrm{e}^{-\Delta_{1}} & \mathrm{e}^{-\Delta_{1}}
\end{array}\right) \\
& =\left(\begin{array}{ll}
\mathrm{e}^{\Delta_{1}}+r_{1} r_{2} \mathrm{e}^{-\Delta_{1}} & r_{1} \mathrm{e}^{-\Delta_{1}}+r_{2} \mathrm{e}^{\Delta_{1}} \\
r_{1} \mathrm{e}^{\Delta_{1}}+r_{2} \mathrm{e}^{-\Delta_{1}} & \mathrm{e}^{-\Delta_{1}}+r_{1} r_{2} \mathrm{e}^{\Delta_{1}}
\end{array}\right) \equiv\left(\begin{array}{ll}
a_{3} & b_{3} \\
c_{3} & d_{3}
\end{array}\right),
\end{aligned}
$$

where $\Delta_{1} \equiv \gamma_{1} d_{1}=\alpha_{1} d_{1}+i \beta_{1} d_{1}$ (see formulae $(21)$ for $m=1$ ) and the Fresnel coefficients $r_{1}$ and $r_{2}$ have the exponential form (19).

The general formulae (29) yield the required expressions for the reflectance and transmittance of the $3 \mathrm{M}$ structure

$$
R=\frac{\left|c_{3}\right|^{2}}{\left|a_{3}\right|^{2}}, \quad T=\frac{n_{2}}{n_{0}} \frac{\left|t_{1}\right|^{2}\left|t_{2}\right|^{2}}{\left|a_{3}\right|^{2}} .
$$

From elements of the coupling matrix (31) and (30), we have obtained

$$
\begin{aligned}
\left|a_{3}\right|^{2}= & \left(\mathrm{e}^{2 \alpha_{1} d_{1}}+\bar{\rho}_{1}^{2} \bar{\rho}_{2}^{2} \mathrm{e}^{-2 \alpha_{1} d_{1}}\right) \\
& +2 \bar{\rho}_{1} \bar{\rho}_{2} \cos \left[\left(2 \beta_{1} d_{1}-\bar{\phi}_{1}\right)-\bar{\phi}_{2}\right] \\
\left|c_{3}\right|^{2}= & \left(\bar{\rho}_{1}^{2} \mathrm{e}^{2 \alpha_{1} d_{1}}+\bar{\rho}_{2}^{2} \mathrm{e}^{-2 \alpha_{1} d_{1}}\right) \\
& +2 \bar{\rho}_{1} \bar{\rho}_{2} \cos \left[\left(2 \beta_{1} d_{1}+\bar{\phi}_{1}\right)-\bar{\phi}_{2}\right] \\
\left|t_{1}\right|^{2}\left|t_{2}\right|^{2}= & \left(1+\bar{\rho}_{1}^{2}+2 \bar{\rho}_{1} \cos \bar{\phi}_{1}\right)\left(1+\bar{\rho}_{2}^{2}+2 \bar{\rho}_{2} \cos \bar{\phi}_{2}\right)
\end{aligned}
$$

where $\bar{\rho}_{1,2} \equiv s_{1,2} \rho_{1,2}$ and $\bar{\phi}_{1,2} \equiv s_{1,2} \phi_{1,2}$.

3.4. Reflectance and Transmittance of the 4M Structure. For the 4M structure shown in Figure 1(b) the coupling matrix product has the form

$$
C_{1} C_{2} C_{3}=\left(\begin{array}{ll}
a_{3} & b_{3} \\
c_{3} & d_{3}
\end{array}\right)\left(\begin{array}{cc}
\mathrm{e}^{\Delta_{2}} & r_{3} \mathrm{e}^{\Delta_{2}} \\
r_{3} \mathrm{e}^{-\Delta_{2}} & \mathrm{e}^{-\Delta_{2}}
\end{array}\right) \equiv\left(\begin{array}{ll}
a_{4} & b_{4} \\
c_{4} & d_{4}
\end{array}\right),
$$

with the elements $a_{4}$ and $c_{4}$ being equal to

$$
\begin{aligned}
& a_{4}=\mathrm{e}^{\left(\Delta_{1}+\Delta_{2}\right)}+r_{1} r_{2} \mathrm{e}^{-\left(\Delta_{1}-\Delta_{2}\right)}+r_{1} r_{3} \mathrm{e}^{-\left(\Delta_{1}+\Delta_{2}\right)}+r_{2} r_{3} \mathrm{e}^{\left(\Delta_{1}-\Delta_{2}\right)}, \\
& c_{4}=r_{1} \mathrm{e}^{\left(\Delta_{1}+\Delta_{2}\right)}+r_{2} \mathrm{e}^{-\left(\Delta_{1}-\Delta_{2}\right)}+r_{3} \mathrm{e}^{-\left(\Delta_{1}+\Delta_{2}\right)}+r_{1} r_{2} r_{3} \mathrm{e}^{\left(\Delta_{1}-\Delta_{2}\right)} .
\end{aligned}
$$

Here, $\Delta_{1,2} \equiv \gamma_{1,2} d_{1,2}=\alpha_{1,2} d_{1,2}+i \beta_{1,2} d_{1,2}$ (see formulae (21) for $m=1,2$ ) and the Fresnel coefficients $r_{m}$ are of exponential form (19), namely, $r_{m}=\bar{\rho}_{m} \exp \left(i \bar{\phi}_{m}\right)$ with $\bar{\rho}_{m}=s_{m} \rho_{m}$ and $\bar{\phi}_{m}=s_{m} \phi_{m}$ (see (11), (15), and (18) for $m=1,2,3)$.

The general formulae (29) yield the required expressions for the reflectance and transmittance of the 4M-structure (cf. expressions (32))

$$
R=\frac{\left|c_{4}\right|^{2}}{\left|a_{4}\right|^{2}}, \quad T=\frac{n_{3}}{n_{0}} \frac{\left|t_{1}\right|^{2}\left|t_{2}\right|^{2}\left|t_{3}\right|^{2}}{\left|a_{4}\right|^{2}},
$$


where the product $\left|t_{1}\right|^{2}\left|t_{2}\right|^{2}\left|t_{3}\right|^{2}$ is written similar to expression (35).

From (37), after some laborious transformations, we obtain the desired expressions

$$
\begin{aligned}
& \left|a_{4}\right|^{2}=\left(\left|a_{3}\right|^{2} \mathrm{e}^{2 \alpha_{2} d_{2}}+\bar{\rho}_{3}^{2}\left|a_{3}^{\prime}\right|^{2} \mathrm{e}^{-2 \alpha_{2} d_{2}}\right)+\bar{\rho}_{3} f_{a}\left(\beta_{2} d_{2}\right), \\
& \left|c_{4}\right|^{2}=\left(\left|c_{3}\right|^{2} \mathrm{e}^{2 \alpha_{2} d_{2}}+\bar{\rho}_{3}^{2}\left|c_{3}^{\prime}\right|^{2} \mathrm{e}^{-2 \alpha_{2} d_{2}}\right)+\bar{\rho}_{3} f_{c}\left(\beta_{2} d_{2}\right),
\end{aligned}
$$

where the terms $\left|a_{3}\right|^{2}$ and $\left|c_{3}\right|^{2}$ have (33) and (34) inherent in the $3 \mathrm{M}$ structure. Besides, (39) include certain additional terms

(i) the terms due to the interference and absorption in layer 1 of optical thickness $\beta_{1} d_{1}$

$$
\begin{aligned}
\left|a_{3}^{\prime}\right|^{2}= & \left(\bar{\rho}_{1}^{2} \mathrm{e}^{-2 \alpha_{1} d_{1}}+\bar{\rho}_{2}^{2} \mathrm{e}^{2 \alpha_{1} d_{1}}\right) \\
& +2 \bar{\rho}_{1} \bar{\rho}_{2} \cos \left[\left(2 \beta_{1} d_{1}-\bar{\phi}_{1}\right)+\bar{\phi}_{2}\right], \\
\left|c_{3}^{\prime}\right|^{2}= & \left(\mathrm{e}^{-2 \alpha_{1} d_{1}}+\bar{\rho}_{1}^{2} \bar{\rho}_{2}^{2} \mathrm{e}^{2 \alpha_{1} d_{1}}\right) \\
& +2 \bar{\rho}_{1} \bar{\rho}_{2} \cos \left[\left(2 \beta_{1} d_{1}+\bar{\phi}_{1}\right)+\bar{\phi}_{2}\right],
\end{aligned}
$$

(ii) the terms due to the interference and absorption in layer 2 of optical thickness $\beta_{2} d_{2}$

$$
\begin{array}{r}
\bar{\rho}_{3} f_{a}\left(\beta_{2} d_{2}\right)=2 \bar{\rho}_{1} \bar{\rho}_{3}\left\{\cos \left[\left(2 \beta_{1} d_{1}-\bar{\phi}_{1}\right)+\left(2 \beta_{2} d_{2}-\bar{\phi}_{3}\right)\right]\right. \\
\left.+\bar{\rho}_{2}^{2} \cos \left[\left(2 \beta_{1} d_{1}-\bar{\phi}_{1}\right)-\left(2 \beta_{2} d_{2}-\bar{\phi}_{3}\right)\right]\right\} \\
+2 \bar{\rho}_{2} \bar{\rho}_{3}\left\{\mathrm{e}^{2 \alpha_{1} d_{1}} \cos \left[\left(2 \beta_{2} d_{2}-\bar{\phi}_{3}\right)-\bar{\phi}_{2}\right]\right. \\
\left.+\bar{\rho}_{1}^{2} \mathrm{e}^{-2 \alpha_{1} d_{1}} \cos \left[\left(2 \beta_{2} d_{2}-\bar{\phi}_{3}\right)+\bar{\phi}_{2}\right]\right\}, \\
\bar{\rho}_{3} f_{c}\left(\beta_{2} d_{2}\right)=2 \bar{\rho}_{1} \bar{\rho}_{3}\left\{\cos \left[\left(2 \beta_{1} d_{1}+\bar{\phi}_{1}\right)+\left(2 \beta_{2} d_{2}-\bar{\phi}_{3}\right)\right]\right. \\
\left.+\bar{\rho}_{2}^{2} \cos \left[\left(2 \beta_{1} d_{1}+\bar{\phi}_{1}\right)-\left(2 \beta_{2} d_{2}-\bar{\phi}_{3}\right)\right]\right\} \\
+2 \bar{\rho}_{2} \bar{\rho}_{3}\left\{\mathrm{e}^{-2 \alpha_{1} d_{1}} \cos \left[\left(2 \beta_{2} d_{2}-\bar{\phi}_{3}\right)+\bar{\phi}_{2}\right]\right. \\
\left.+\bar{\rho}_{1}^{2} \mathrm{e}^{2 \alpha_{1} d_{1}} \cos \left[\left(2 \beta_{2} d_{2}-\bar{\phi}_{3}\right)-\bar{\phi}_{2}\right]\right\} .
\end{array}
$$

From a comparison of (33)-(34) and (40), it follows that both terms $\left(\left|a_{3}\right|^{2},\left|c_{3}\right|^{2}\right)$ and $\left(\left|a_{3}^{\prime}\right|^{2},\left|c_{3}^{\prime}\right|^{2}\right)$ are produced by the interference and absorption phenomena only inside layer 1 . Both of them, being inserted in the $4 \mathrm{M}$ composition $\left(\left|a_{4}\right|^{2},\left|c_{4}\right|^{2}\right)$, as seen from (39), prove to be multiplied by the factors $\exp \left( \pm 2 \alpha_{2} d_{2}\right)$ due to optical losses in layer 2 . The interference oscillations in this layer (together with an additional contribution from interference and absorption in layer 1$)$ are solely taken into account by the functions $\bar{\rho}_{3} f_{a}\left(\beta_{2} d_{2}\right)$ and $\bar{\rho}_{3} f_{c}\left(\beta_{2} d_{2}\right)$ given in the form of $(41)$.

It is very important to note that both the additional terms $\left(\left|a_{3}^{\prime}\right|^{2},\left|c_{3}^{\prime}\right|^{2}\right)$ and $\left(f_{a}, f_{c}\right)$ contribute to expressions (39) for the $4 \mathrm{M}$ structure being multiplied by $\rho_{3} \equiv\left|r_{3}\right| \propto\left|\tilde{n}_{2}-\tilde{n}_{3}\right|$. Therefore, their contributions to (38) for $R$ and $T$ disappear and, as a result of this, they are converted into (32) for the $3 \mathrm{M}$ structure when

$$
\tilde{n}_{3}=\tilde{n}_{2}, \quad d_{2}=0 .
$$

Both requirements (42) are needed for the conversion $4 \mathrm{M} \rightarrow 3 \mathrm{M}$, as applied to the transmittance $T$, while the reflectance conversion is immediately fulfilled if $\tilde{n}_{3}=\tilde{n}_{2}$, that is, if only medium 3 does not differ from medium 2 .

Hence, our further transformations will deal with the $4 \mathrm{M}$ structure, as a general one, to reduce the reflectance $R$ and transmittance $T$ given by (38)-(41) to the so-called physical form. Such a form has to contain instead of the Fresnel coefficients the physical constants of media (refractive and absorptive indices) as well as the geometrical parameters of a structure under consideration.

\section{Physical Form of Expressions for the Reflectance and Transmittance}

4.1. General Expressions. Appendix A is devoted to expressing the quantities $\left|a_{4}\right|^{2}$ and $\left|c_{4}\right|^{2}$ obtained above in the form of (39)-(41) in terms of the physical and geometrical parameters of the $4 \mathrm{M}$ structure. The final result is given by relations (A.39)-(A.40) and so their substitution into (38) yields the desired expressions for the reflectance and transmittance

$$
\begin{gathered}
R=\frac{L_{-}+M \cos \left(2 \beta_{1} d_{1}-\varphi_{-}\right)+F_{-}\left(\beta_{2} d_{2}\right)}{L_{+}+M \cos \left(2 \beta_{1} d_{1}-\varphi_{+}\right)+F_{+}\left(\beta_{2} d_{2}\right)}, \\
T=\frac{16 n_{0}\left|\tilde{n}_{1}\right|^{2}\left|\tilde{n}_{2}\right|^{2} n_{3}}{L_{+}+M \cos \left(2 \beta_{1} d_{1}-\varphi_{+}\right)+F_{+}\left(\beta_{2} d_{2}\right)},
\end{gathered}
$$

where all the subscripts + and - are in line with double signs appearing in the proper coefficients given below.

In (43), we have introduced the following new quantities:

(i) the amplitude $M$ and phase angles $\varphi_{ \pm}$of the interference oscillations in layer 1 of the optical thickness $\beta_{1} d_{1}$ (see (A.14)-(A.16) and (A.18)-(A.19))

$$
\begin{aligned}
& M= s_{1} s_{2} S_{12} \sqrt{\left(A \cos \bar{\phi}_{2}\right)^{2}+\left(B \sin \bar{\phi}_{2}\right)^{2}}, \\
& \varphi_{ \pm}=\psi \pm \bar{\phi}_{1} \quad \text { with } \tan \psi=\left(\frac{B}{A}\right) \tan \bar{\phi}_{2}, \\
& A=\left(\left|\tilde{n}_{2}\right|^{2}+\left|\tilde{n}_{3}\right|^{2}\right) \cosh 2 \alpha_{2} d_{2} \\
&+2\left(n_{2} n_{3}+k_{2} k_{3}\right) \sinh 2 \alpha_{2} d_{2}, \\
& B=\left(\left|\tilde{n}_{2}\right|^{2}+\left|\tilde{n}_{3}\right|^{2}\right) \sinh 2 \alpha_{2} d_{2} \\
&+2\left(n_{2} n_{3}+k_{2} k_{3}\right) \cosh 2 \alpha_{2} d_{2}, \\
& S_{12}=\left\{\left[\left(n_{0}-n_{1}\right)^{2}+k_{1}^{2}\right]\left[\left(n_{0}+n_{1}\right)^{2}+k_{1}^{2}\right]\right. \\
& \quad \times\left[\left(n_{1}-n_{2}\right)^{2}+\left(k_{1}-k_{2}\right)^{2}\right] \\
& \times {\left.\left[\left(n_{1}+n_{2}\right)^{2}+\left(k_{1}+k_{2}\right)^{2}\right]\right\}^{1 / 2}, }
\end{aligned}
$$


(ii) the losses parameters $L_{ \pm}$due to optical absorption (see (A.26)-(A.27))

$$
\begin{aligned}
& L_{ \pm}=a_{ \pm} \cosh 2 \alpha_{1} d_{1}+b_{ \pm} \sinh 2 \alpha_{1} d_{1} \\
& a_{ \pm}=\left(n_{0}^{2}+\left|\tilde{n}_{1}\right|^{2}\right)\left(\left|\tilde{n}_{1}\right|^{2}+\left|\tilde{n}_{2}\right|^{2}\right) A \\
& \pm 4 n_{0} n_{1}\left(n_{1} n_{2}+k_{1} k_{2}\right) B \\
& b_{ \pm}=2\left[\left(n_{0}^{2}+\left|\tilde{n}_{1}\right|^{2}\right)\left(n_{1} n_{2}+k_{1} k_{2}\right) B\right. \\
& \left. \pm n_{0} n_{1}\left(\left|\tilde{n}_{1}\right|^{2}+\left|\tilde{n}_{2}\right|^{2}\right) A\right] \text {, }
\end{aligned}
$$

(iii) the functions $F_{ \pm}\left(\beta_{2} d_{2}\right)$ taking into account the interference oscillations in layer 2 of the optical thickness $\beta_{2} d_{2}$ (together with an additional contribution from the interference and absorption in layer 1) (see (A.32)-(A.33) and (A.36)-(A.38))

$$
\begin{aligned}
& F_{ \pm}\left(\beta_{2} d_{2}\right)=N_{ \pm} \cos \left[\left(2 \beta_{2} d_{2}-\bar{\phi}_{3}\right)-\psi_{ \pm}\right] \\
& +P \cos \left(2 \beta_{1} d_{1} \mp \bar{\phi}_{1}\right) \cos \left(2 \beta_{2} d_{2}-\bar{\phi}_{3}\right) \\
& -Q \sin \left(2 \beta_{1} d_{1} \mp \bar{\phi}_{1}\right) \sin \left(2 \beta_{2} d_{2}-\bar{\phi}_{3}\right) \text {, } \\
& N_{ \pm}=s_{2} s_{3} S_{23} \sqrt{\left(A_{ \pm} \cos \bar{\phi}_{2}\right)^{2}+\left(B_{ \pm} \sin \bar{\phi}_{2}\right)^{2}} \\
& \tan \psi_{ \pm}=\left(\frac{B_{ \pm}}{A_{ \pm}}\right) \tan \bar{\phi}_{2} \\
& A_{ \pm}=\left(n_{0}^{2}+\left|\tilde{n}_{1}\right|^{2}\right) \cosh 2 \alpha_{1} d_{1} \pm 2 n_{0} n_{1} \sinh 2 \alpha_{1} d_{1} \\
& B_{ \pm}=\left(n_{0}^{2}+\left|\tilde{n}_{1}\right|^{2}\right) \sinh 2 \alpha_{1} d_{1} \pm 2 n_{0} n_{1} \cosh 2 \alpha_{1} d_{1} \\
& P=\left(\left|\tilde{n}_{1}\right|^{2}+\left|\tilde{n}_{2}\right|^{2}\right) s_{1} s_{3} S_{13}, \quad Q=2\left(n_{1} n_{2}+k_{1} k_{2}\right) s_{1} s_{3} S_{13}, \\
& S_{13}=\left\{\left[\left(n_{0}-n_{1}\right)^{2}+k_{0}^{2}\right]\left[\left(n_{0}+n_{1}\right)^{2}+k_{0}^{2}\right]\right. \\
& \times\left[\left(n_{2}-n_{3}\right)^{2}+\left(k_{2}-k_{3}\right)^{2}\right] \\
& \left.\times\left[\left(n_{2}+n_{3}\right)^{2}+\left(k_{2}+k_{3}\right)^{2}\right]\right\}^{1 / 2} \text {, } \\
& S_{23}=\left\{\left[\left(n_{1}-n_{2}\right)^{2}+\left(k_{1}-k_{2}\right)^{2}\right]\left[\left(n_{1}+n_{2}\right)^{2}+\left(k_{1}+k_{2}\right)^{2}\right]\right. \\
& \times\left[\left(n_{2}-n_{3}\right)^{2}+\left(k_{2}-k_{3}\right)^{2}\right] \\
& \left.\times\left[\left(n_{2}+n_{3}\right)^{2}+\left(k_{2}+k_{3}\right)^{2}\right]\right\}^{1 / 2} \text {. }
\end{aligned}
$$

The first term in (52) for $F_{ \pm}\left(\beta_{2} d_{2}\right)$ is a contribution caused by single interference oscillations in layer 2 having the amplitudes $N_{ \pm}$and phase angles $\psi_{ \pm}$, which is similar to the interference term of layer 1 with the amplitude $M$ and phase angles $\varphi_{ \pm}$. The last two terms in (52) involving products of trigonometric functions with the amplitudes $P$ and $Q$ reflect a contribution from the double (intermodulation) interference due to mutual oscillations in both layers 1 and 2 .

4.2. Particular Expressions Taking Account of the Device Averaging. It is the functions $F_{ \pm}\left(\beta_{2} d_{2}\right)$ that take into account the fast oscillations generated by interference in a thick substrate (when $n_{2} d_{2} \gg n_{1} d_{1}$ ) and result in appearing a "beard" on the optical spectra similar to that shown in Figure 2. As discussed above, any spectrophotometer with a operating slit of finite width cannot register these fast oscillations. In other words, such a spectrophotometer performs the socalled device averaging whose mathematical justification is given in Appendix B. As follows from (B.11) and (B.12), the device averaging allows us to assume $F_{ \pm}\left(\beta_{2} d_{2}\right) \sim 0$ in (43) for the spectra $R(\lambda)$ and $T(\lambda)$.

Thus, when taking into account the device averaging, (43) for the 4M structure take the simplest and succinct form

$$
\begin{aligned}
& R=\frac{L_{-}+M \cos \left(2 \beta_{1} d_{1}-\varphi_{-}\right)}{L_{+}+M \cos \left(2 \beta_{1} d_{1}-\varphi_{+}\right)}, \\
& T=\frac{16 n_{0}\left|\tilde{n}_{1}\right|^{2}\left|\tilde{n}_{2}\right|^{2} n_{3}}{L_{+}+M \cos \left(2 \beta_{1} d_{1}-\varphi_{+}\right)},
\end{aligned}
$$

with all the quantities (44)-(51) being left unchanged.

Accordingly, by means of these quantities the spectra $R(\lambda)$ and $T(\lambda)$ now take into account the optical constants $\left(n_{2}, k_{2}\right)$ of a thick substrate and its finite thickness $\left(d_{2} \gg d_{1}\right)$ but fully disregard interference effects in the substrate.

The analogous simplified form of $R$ and $T$, as applied to the special case of the $3 \mathrm{M}$ structure, follows immediately from (43). Indeed, if (42) of the conversion $4 \mathrm{M} \rightarrow 3 \mathrm{M}$ are fulfilled, (58) and (59) provide $S_{13}=S_{23}=0$ (because of $n_{3}=n_{2}$ and $\left.k_{3}=k_{2}\right)$ so that $F_{ \pm}\left(\beta_{2} d_{2}\right)=0$ because of $N_{ \pm}=P=Q=0$. Besides, in this case from (46) and (47) it follows that

$$
A=B=2\left|\tilde{n}_{2}\right|^{2}
$$

which ensures two consequences: (i) the phase angles $\varphi_{ \pm}=$ $\bar{\phi}_{2} \pm \bar{\phi}_{1}$ since $\tan \psi=\tan \bar{\phi}_{2}$ when $A=B$ (see (45)), (ii) the quantities $M$ and $L_{ \pm}$get a common multiplier equal to (62) (see (44) and (49)-(51)), which is cancelled after substituting into the numerator and denominator of (43) for $R$ and $T$.

In such a case, (43) assume the forms specific to the $3 \mathrm{M}$ structure of arbitrary physical and geometrical properties

$$
\begin{aligned}
& R=\frac{L_{-}+M \cos \left(2 \beta_{1} d_{1}-\varphi_{-}\right)}{L_{+}+M \cos \left(2 \beta_{1} d_{1}-\varphi_{+}\right)}, \\
& T=\frac{8 n_{0}\left|\tilde{n}_{1}\right|^{2} n_{2}}{L_{+}+M \cos \left(2 \beta_{1} d_{1}-\varphi_{+}\right)},
\end{aligned}
$$


where (cf. (44), (45), (45) and (49)-(51))

$$
\begin{gathered}
M=s_{1} s_{2} S_{12}, \quad \varphi_{ \pm}=\bar{\phi}_{2} \pm \bar{\phi}_{1}, \\
S_{12}=\left\{\left[\left(n_{0}-n_{1}\right)^{2}+k_{1}^{2}\right]\left[\left(n_{0}+n_{1}\right)^{2}+k_{1}^{2}\right]\right. \\
\times\left[\left(n_{1}-n_{2}\right)^{2}+\left(k_{1}-k_{2}\right)^{2}\right] \\
\left.\times\left[\left(n_{1}+n_{2}\right)^{2}+\left(k_{1}+k_{2}\right)^{2}\right]\right\}^{1 / 2}, \\
L_{ \pm}=a_{ \pm} \cosh 2 \alpha_{1} d_{1}+b_{ \pm} \sinh 2 \alpha_{1} d_{1}, \\
a_{ \pm}=\left(n_{0}^{2}+\left|\tilde{n}_{1}\right|^{2}\right)\left(\left|\tilde{n}_{1}\right|^{2}+\left|\tilde{n}_{2}\right|^{2}\right) \pm 4 n_{0} n_{1}\left(n_{1} n_{2}+k_{1} k_{2}\right),
\end{gathered}
$$

$b_{ \pm}=2\left[\left(n_{0}^{2}+\left|\tilde{n}_{1}\right|^{2}\right)\left(n_{1} n_{2}+k_{1} k_{2}\right) \pm n_{0} n_{1}\left(\left|\tilde{n}_{1}\right|^{2}+\left|\tilde{n}_{2}\right|^{2}\right)\right]$.

Expressions (63)-(69) are valid for any one of the $3 \mathrm{M}$ structures and give appropriate equations for the different special cases encountered in the literature [1-12].

The angles $\bar{\phi}_{1}$ and $\bar{\phi}_{2}$ appearing in (45) and (65) display a phase of the Fresnel reflection coefficients (19) for interfaces 1 and 2 (shown in Figure 1(b)) and, in accordance with formulae (A.2) and (A.3), they are defined in the form

$$
\begin{gathered}
\tan \bar{\phi}_{1} \equiv \tan \left(s_{1} \phi_{1}\right)=s_{1} \tan \phi_{1}=\frac{2 n_{0} k_{1}}{n_{0}^{2}-\left|\tilde{n}_{1}\right|^{2}} \\
\tan \bar{\phi}_{2} \equiv \tan \left(s_{2} \phi_{2}\right)=s_{2} \tan \phi_{2}=\frac{2\left(n_{1} k_{2}-n_{2} k_{1}\right)}{\left|\tilde{n}_{1}\right|^{2}-\left|\tilde{n}_{2}\right|^{2}} .
\end{gathered}
$$

As follows from (70), these angles may be called the losses angles because in a lossless situation they vanish $\tan \bar{\phi}_{1}=$ $\tan \bar{\phi}_{2}=0$ when $k_{1}=k_{2}=0$.

It is extremely remarkable that (60)-(61) for the $4 \mathrm{M}$ structures with the device averaging and expressions (63)(64) for the arbitrary 3M structures completely coincide in form and differ only by the appropriate parameters (44)(51) for the former and (65)-(69) for the latter, which are also of the same configuration. Such a coincidence enable the reflectance and/or transmission spectra to be analyzed below in the same way, as applied to both types of the film-substrate structures.

There is a variety of the $3 \mathrm{M}$ structures and the $4 \mathrm{M}$ structures but only two of them find the wide application in optical measurement techniques, namely

(i) an arbitrary film on a highly absorbing (metal) substrate

(ii) a slightly absorbing film on a transparent (dielectric) substrate.

Let us consider modifications of the above expressions for $R$ and $T$, as applied to the two particular cases.
4.2.1. Arbitrary Films on Highly Absorbing Substrates. are characterized by the only requirement

$\alpha_{2} d_{2}$ is so much large that

$$
\cosh 2 \alpha_{2} d_{2} \simeq \sinh 2 \alpha_{2} d_{2} \simeq \frac{1}{2} \mathrm{e}^{2 \alpha_{2} d_{2}} .
$$

Then, from (46) and (47), it follows that

$$
A=B=\frac{1}{2}\left[\left(n_{2}+n_{3}\right)^{2}+\left(k_{2}+k_{3}\right)^{2}\right] \mathrm{e}^{2 \alpha_{2} d_{2}} .
$$

The condition (72) works as well as (62) did before; that is, it ensures the same consequences: (i) the phase angles $\varphi_{ \pm}=$ $\bar{\phi}_{2} \pm \bar{\phi}_{1}$ because $\tan \psi=\tan \bar{\phi}_{2}$ when $A=B$ (see (45)) and (ii) the quantities $M$ and $L_{ \pm}$acquire a common multiplier equal to (72) (see (44) and (49)-(51)).

Hence, the reflectance $R$ of the $4 \mathrm{M}$ structure with a highly absorbing substrate, provided (71) holds true, is described by (63) inherent in the $3 \mathrm{M}$ structure with (65)-(70) left unchanged. As for the transmittance $T$, its expression (61) proves to be divided by (72) so that values of $T$ practically vanish because of the factor $\exp \left(-2 \alpha_{2} d_{2}\right) \rightarrow 0$. Therefore, for thin films on a highly absorbing substrates, there is no point in using formulae for the $4 \mathrm{M}$ structure.

4.2.2. Slightly Absorbing Films on Transparent Substrates. are characterized by the following requirements:

$$
\begin{gathered}
k_{1}^{2} \ll n_{1}^{2}, \quad\left|\tilde{n}_{1}\right|^{2} \simeq n_{1}^{2}, \\
k_{2}=k_{3}=\alpha_{2} d_{2}=0, \quad\left|\tilde{n}_{2}\right|^{2}=n_{2}^{2} \quad\left|\tilde{n}_{3}\right|^{2}=n_{3}^{2} .
\end{gathered}
$$

Then, from (46)-(47) and (48) or (66), it, respectively, follows that

$$
A=n_{2}^{2}+n_{3}^{2}, \quad B=2 n_{2} n_{3},
$$

$S_{12}=\left|n_{0}^{2}-n_{1}^{2}\right|\left|n_{1}^{2}-n_{2}^{2}\right|, \quad s_{1} s_{2} S_{12}=\left(n_{0}^{2}-n_{1}^{2}\right)\left(n_{1}^{2}-n_{2}^{2}\right)$.

Requirements (73) modify the losses angles $\bar{\phi}_{1}$ and $\bar{\phi}_{2}$ so that (70) take the following small-losses form

$$
\tan \bar{\phi}_{1} \simeq \frac{2 n_{0} k_{1}}{n_{0}^{2}-n_{1}^{2}}, \quad \tan \bar{\phi}_{2} \simeq-\frac{2 n_{2} k_{1}}{n_{1}^{2}-n_{2}^{2}} .
$$

In terms of (74) and (76), (45) assumes the form

$$
\tan \psi=\frac{B}{A} \tan \bar{\phi}_{2} \simeq-\frac{4 n_{2}^{2} n_{3} k_{1}}{\left(n_{1}^{2}-n_{2}^{2}\right)\left(n_{2}^{2}+n_{3}^{2}\right)} .
$$

Formulae (74)-(77) are used below to obtain some simplified expressions for the basic quantities $M, \varphi_{ \pm}$and $L_{ \pm}$defining the reflectance and transmission spectra. In so doing, we allow for the small-losses consequences $\left(\tan \bar{\phi}_{2}\right)^{2} \ll 1$ and $(\tan \psi)^{2} \ll 1$.

The amplitude $M$ of interference oscillations follows from formulae (65) and (44) in the following form:

(i) for the $3 \mathrm{M}$ structure

$$
M=s_{1} s_{2} S_{12}=\left(n_{0}^{2}-n_{1}^{2}\right)\left(n_{1}^{2}-n_{2}^{2}\right),
$$

(ii) for the $4 \mathrm{M}$ structure

$$
M \simeq s_{1} s_{2} S_{12} A=\left(n_{0}^{2}-n_{1}^{2}\right)\left(n_{1}^{2}-n_{2}^{2}\right)\left(n_{2}^{2}+n_{3}^{2}\right) .
$$


The phase angles $\varphi_{ \pm}$of interference oscillations for the $3 \mathrm{M}$ and $4 \mathrm{M}$ structures given by (65) and (45) can be represented in a common form

$$
\tan \varphi_{ \pm}=\frac{m_{ \pm}}{M} \frac{k_{1}}{n_{1}}
$$

where

(i) for the $3 \mathrm{M}$ structure

$$
m_{ \pm}=2 n_{1}\left[-\left(n_{0}^{2}-n_{1}^{2}\right) n_{2} \pm n_{0}\left(n_{1}^{2}-n_{2}^{2}\right)\right]
$$

(ii) for the $4 \mathrm{M}$ structure

$$
\begin{aligned}
m_{ \pm} & =2 n_{1}\left[-\left(n_{0}^{2}-n_{1}^{2}\right) n_{2} B \pm n_{0}\left(n_{1}^{2}-n_{2}^{2}\right) A\right] \\
& =2 n_{1}\left[-2\left(n_{0}^{2}-n_{1}^{2}\right) n_{2}^{2} n_{3} \pm n_{0}\left(n_{1}^{2}-n_{2}^{2}\right)\left(n_{2}^{2}+n_{3}^{2}\right)\right] .
\end{aligned}
$$

The losses parameters $L_{ \pm}$for the $3 \mathrm{M}$ and $4 \mathrm{M}$ structures retain the common form (67) or (49) with the factors $a_{ \pm}$and $b_{ \pm}$of the following form:

(i) for the $3 \mathrm{M}$ structure

$$
\begin{gathered}
a_{ \pm}=\left(n_{0}^{2}+n_{1}^{2}\right)\left(n_{1}^{2}+n_{2}^{2}\right) \pm 4 n_{0} n_{1}^{2} n_{2}, \\
b_{ \pm}=2 n_{1}\left[\left(n_{0}^{2}+n_{1}^{2}\right) n_{2} \pm n_{0}\left(n_{1}^{2}+n_{2}^{2}\right)\right],
\end{gathered}
$$

(ii) for the $4 \mathrm{M}$ structure

$$
\begin{aligned}
a_{ \pm} & =\left(n_{0}^{2}+n_{1}^{2}\right)\left(n_{1}^{2}+n_{2}^{2}\right) A \pm 4 n_{0} n_{1}^{2} n_{2} B \\
& =\left(n_{0}^{2}+n_{1}^{2}\right)\left(n_{1}^{2}+n_{2}^{2}\right)\left(n_{2}^{2}+n_{3}^{2}\right) \pm 8 n_{0} n_{1}^{2} n_{2}^{2} n_{3}, \\
b_{ \pm} & =2 n_{1}\left[\left(n_{0}^{2}+n_{1}^{2}\right) n_{2} B \pm n_{0}\left(n_{1}^{2}+n_{2}^{2}\right) A\right] \\
& =2 n_{1}\left[2\left(n_{0}^{2}+n_{1}^{2}\right) n_{2}^{2} n_{3} \pm n_{0}\left(n_{1}^{2}+n_{2}^{2}\right)\left(n_{2}^{2}+n_{3}^{2}\right)\right] .
\end{aligned}
$$

Let us analyze the reflectance and transmittance spectra of the $4 \mathrm{M}$ structure with the device averaging and the $3 \mathrm{M}$ structure by taking advantage of coinciding with (60)-(61) for the former and with (63)-(64) for the latter.

\section{Analysis of the Reflectance and Transmittance Spectra}

All the relations obtained above are valid for the general case when each $m$ th medium incorporated into the film-substrate structure is absorbing and dispersive, that is, $\tilde{n}_{m}(\lambda)=$ $n_{m}(\lambda)-i k_{m}(\lambda), m=1,2,3$. Such a dependence of the refractive indices on light wavelength $\lambda$ naturally manifests itself in the optical spectra $R(\lambda)$ and $T(\lambda)$. However, a leading contribution into interference oscillations of the spectra is made by the periodic functions $\cos \left(2 \beta_{1} d_{1}-\varphi_{ \pm}\right)$appearing in (60)-(61) or (63)-(64) because usually the functions $\tilde{n}_{m}(\lambda)$ are slowly varying as compared with the periodic functions.

For this reason, below we take into account only the main contribution to the dependencies $R(\lambda)$ and $T(\lambda)$ from the cosine functions.
5.1. Reflectance Spectrum. Let us write down the reflectance spectrum (60) or (63) in the following form:

$$
R(x)=\frac{L_{-}+M \cos \left(2 x-\varphi_{-}\right)}{L_{+}+M \cos \left(2 x-\varphi_{+}\right)} .
$$

We consider instead of $R(\lambda)$ the frequency spectrum $R(x)$ where $x \equiv \beta_{1} d_{1}=(\omega / c) n_{1} d_{1}$ is the dimensionless frequency.

Positions of maxima and minima in the spectrum (85) are found from the condition $\partial R(x) / \partial x=0$ which gives the following transcendental equation to find them:

$$
L_{-} \sin \left(2 \beta_{1} d_{1}-\varphi_{+}\right)-L_{+} \sin \left(2 \beta_{1} d_{1}-\varphi_{-}\right)=M \sin \left(\varphi_{+}-\varphi_{-}\right) .
$$

Here, the quantities $M, \varphi_{ \pm}$and $L_{ \pm}$have different expressions for either the $4 \mathrm{M}$ structure (with the device averaging) or the $3 \mathrm{M}$ structure, namely, they are (44)-(48) and (49)-(51) for the former or (65)-(66) and (67)-(69) for the latter.

An essential simplification of (86) takes place in the particular case of practical interest when a transparent film $\left(k_{1}=0, \alpha_{1} d_{1}=(2 \pi / \lambda) k_{1} d_{1}=0\right)$ is situated on a highly absorbing substrate being subject to the requirement (71). As proved above, for such a physical situation the $3 \mathrm{M}$ structure model is workable even if the substrate is of finite thickness $d_{2}$ because values of $\alpha_{2} d_{2}=(2 \pi / \lambda) k_{2} d_{2}$ are sufficiently large.

In this simplified case, from (70), it follows that the losses angles of interfaces 1 and 2 are equal to

$$
\bar{\phi}_{1}=0, \quad \bar{\phi}_{2}=\arctan \frac{2 n_{1} k_{2}}{n_{1}^{2}-\left|\tilde{n}_{2}\right|^{2}} .
$$

Hence, the phase angles $\varphi_{+}$and $\varphi_{-}$given by (65) as $\varphi_{ \pm}=$ $\bar{\phi}_{2} \pm \bar{\phi}_{1}$ become equal to each other owing to (87), namely,

$$
\varphi \equiv \varphi_{+}=\varphi_{-}=\bar{\phi}_{2}, \quad \tan \varphi=\frac{2 n_{1} k_{2}}{n_{1}^{2}-\left|\tilde{n}_{2}\right|^{2}}
$$

where $|\tan \varphi|$ is not small because of noticeable losses in a highly absorbing substrate. form

Then, (85) for the reflectance assumes the simplified

$$
R(\lambda)=\frac{L_{-}+M \cos \left(2 \beta_{1} d_{1}-\varphi\right)}{L_{+}+M \cos \left(2 \beta_{1} d_{1}-\varphi\right)},
$$

where the quantities $M$ and $L_{ \pm}$generally given by (65)(69) are also reduced with taking into account (88) to the following simplified form:

$$
\begin{gathered}
M=s_{1} s_{2} S_{12}=\left(n_{0}^{2}-n_{1}^{2}\right)\left(n_{1}^{2}-\left|\tilde{n}_{2}\right|^{2}\right) \sqrt{1+\tan ^{2} \varphi}, \\
L_{ \pm}=a_{ \pm}=\left(n_{0}^{2}+n_{1}^{2}\right)\left(n_{1}^{2}+\left|\tilde{n}_{2}\right|^{2}\right) \pm 4 n_{0} n_{1}^{2} n_{2} .
\end{gathered}
$$

Formulae (88)-(90) have been used to compute the reflectance spectrum $R(\lambda)$ for a non-absorbing film $\left(n_{0}=\right.$ $1, n_{1}=2.3, k_{1}=0$ ) of thickness $d_{1}=1 \mu \mathrm{m}$ deposited on a titanium substrate $\left(n_{2}=1.89, k_{2}=2.58\right)$. Result of the computation is shown in Figure 5 by a solid line. For comparison, here, there is also a dashed line corresponding 


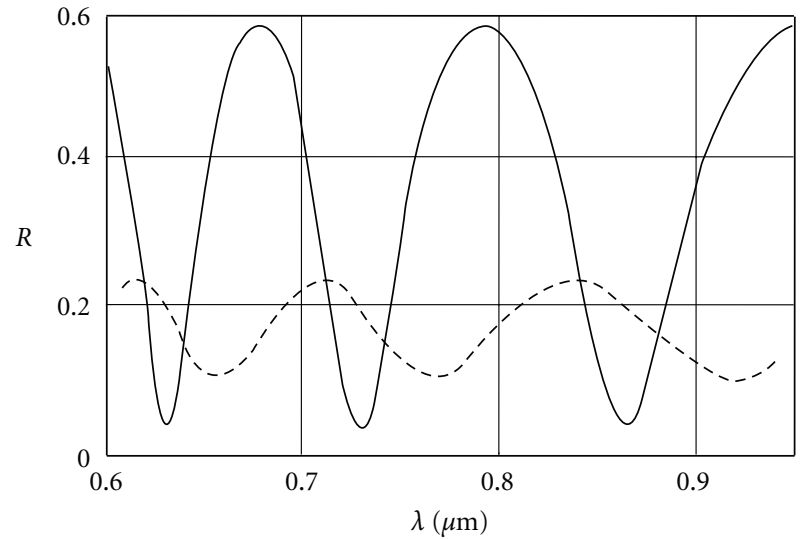

FIGURE 5: Reflectance spectrum of a transparent film on a metal substrate (solid line) and on a nonabsorbing substrate (dashed line).

to the same film on a transparent substrate $\left(n_{2}=1.89, k_{2}=\right.$ $0)$.

As seen from Figure 5, the high absorption of a metal substrate not only influences the amplitude of interference oscillations but also changes the positions of maxima and minima in the spectrum $R(\lambda)$. To find them, it is necessary to apply an equation resulting from (86) as a particular case for which $\varphi_{+}=\varphi_{-}$and having the utterly simplified form

$$
\sin \left(2 \beta_{1} d_{1}-\varphi\right)=0 .
$$

As follows from (91), the absorption of a substrate indeed shifts the spectrum $R(x)$ along the frequency axis $x \equiv \beta_{1} d_{1}=$ $(\omega / c) n_{1} d_{1}$ by the same value for all the maxima and minima equal to the losses angle $\varphi=\bar{\phi}_{2}$.

According to our experimental data, for amorphous oxide films $\mathrm{TiO}_{2}$ effect of the dispersion function $n_{1}(\lambda)$ becomes noticeable for $\lambda<600 \mathrm{~nm}$. Figure 6(a) contains the curve $n_{1}(\lambda)$ which is drawn on the basis of experimental tabular data taken from [27]. We have approximated this curve by an expression (proposed in [24])

$$
n_{1}(\lambda)=\frac{a}{\lambda^{b}}+c
$$

and found its coefficients $a=0.015, b=4.025, c=2.2$ by the least-squares method. The spectrum $R(\lambda)$ for such a dispersive film of thickness $d_{1}=1 \mu \mathrm{m}$ on a titanium substrate $\left(n_{2}=1.89, k_{2}=2.58\right)$ is computed by using (88)(90) and shown in Figure 6(b) by solid line. A dashed curve describes the analogous spectrum calculated from the same formulae for a constant value of $n_{1}=2.3$. From comparison of the two curves, it follows that an increase in $n_{1}(\lambda)$ at short wavelengths leads to a rise in number of oscillations for both the spectra.

5.2. Transmittance Spectrum. The transmittance spectra (61) for the $4 \mathrm{M}$ structure with the device averaging and (64) for the $3 \mathrm{M}$ structure can be represented in a common form

$$
T(\lambda)=\frac{K}{L_{+}+M \cos \left(2 \beta_{1} d_{1}-\varphi_{+}\right)},
$$

where the quantities $M, \varphi_{+}$and $L_{+}$are the same as those in the denominator of $R(\lambda)$ and the numerator $K$ is equal to

(i) for the $3 \mathrm{M}$ structure

$$
K=8 n_{0}\left|\tilde{n}_{1}\right|^{2} n_{2}
$$

(ii) for the $4 \mathrm{M}$ structure

$$
K=16 n_{0}\left|\tilde{n}_{1}\right|^{2}\left|\tilde{n}_{2}\right|^{2} n_{3}
$$

Position of maxima and minima in the spectrum (93) is found from the condition $\partial T(x) / \partial x=0$ (with $x=\beta_{1} d_{1}$ ) which gives the following equation to find them:

$$
\sin \left(2 \beta_{1} d_{1}-\varphi_{+}\right)=0 .
$$

This equations is identical to (91) for $R(x)$ applicable only to transparent films on a highly absorbing substrate whereas (96) for $T(x)$ holds valid always independently of the physical properties and type $(3 \mathrm{M}$ or $4 \mathrm{M})$ of a structure. As follows from (96), the position of maxima and minima in the transmission spectrum is solely controlled by the phase angle $\varphi_{+}$given by (45) or (65).

As noted above, among various thin-film structures, the most practical interest in the transmittance spectrum measurements has been displayed for the transparent or slightly absorbing films on non-absorbing substrates which satisfy (73). The simplified expressions for $M, \varphi_{+}$and $L_{+}$which are applicable to such a kind of the $3 \mathrm{M}$ and $4 \mathrm{M}$ structures have the form given by (78)-(84). These expressions were used to compute the spectrum $T(\lambda)$ of a slightly absorbing film on a transparent substrate with the following parameters: $n_{0}=n_{3}=1, n_{1}=2.21, k_{1}=0.01$, $n_{2}=1.472, k_{2}=0$ and $d_{1}=1 \mu \mathrm{m}$.

The results of calculation are given in Figure 7 as two curves corresponding to both the $3 \mathrm{M}$ structure (solid thick line) and the $4 \mathrm{M}$ structure (dashed line). A comparison of these curves demonstrates that the $3 \mathrm{M}$ structure model with a semiinfinite substrate of index $n_{2}$ (see Figure 1(a)) yields substantially higher values of $T(\lambda)$ because of neglecting influence of medium 3 with index $n_{3}$ and so that of light reflection from interface 3 (see Figure $1(\mathrm{~b})$ ). Besides, there is a solid thin curve calculated by using the extremely simplified (1) and (3) of Swanepoel's approach [24, 25]. These formulae give also higher values of $T(\lambda)$ only at points of spectral minimum, as compared with our formulae derived on the basis of a rigorous theoretical approach. Such a difference in minimum points of $T(\lambda)$ between Swanepoel's and our results amounts to $2.5 \%-3 \%$. This fact refutes a statement of Swanepoel that his theory provides an accuracy error less than $1 \%$.

In order to verify applicability of our formulae to processing of experimental spectra for finding thin-film parameters, a tantalum oxide film was deposited on the quartz-glass substrate $\left(n_{2}=1.472, k_{2}=0, d_{2}=500 \mu \mathrm{m}\right)$ by using the reactive magnetron sputtering method. The experimental spectrum $T(\lambda)$ of such a thin-film structure measured by a spectrophotometer is shown in Figure 8 as a 


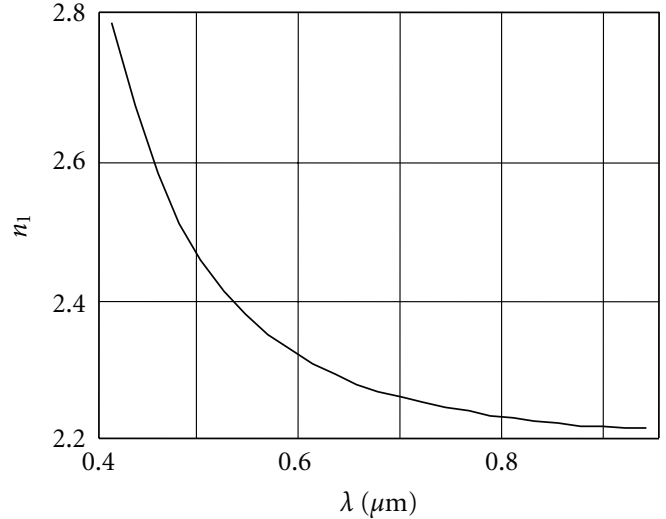

(a)

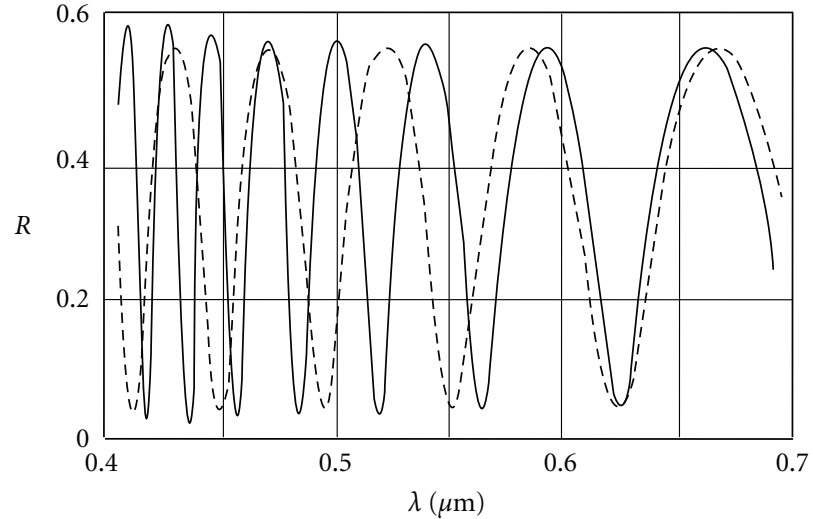

(b)

Figure 6: (a) Experimental curve $n_{1}(\lambda)$ for an oxide film $\mathrm{TiO}_{2}$ [27] and (b) the reflectance spectrum $R(\lambda)$ calculated for such a film (solid line) and for a nondispersive film with $n_{1}=2.3$ (dashed line) both deposited on a titanium substrate.

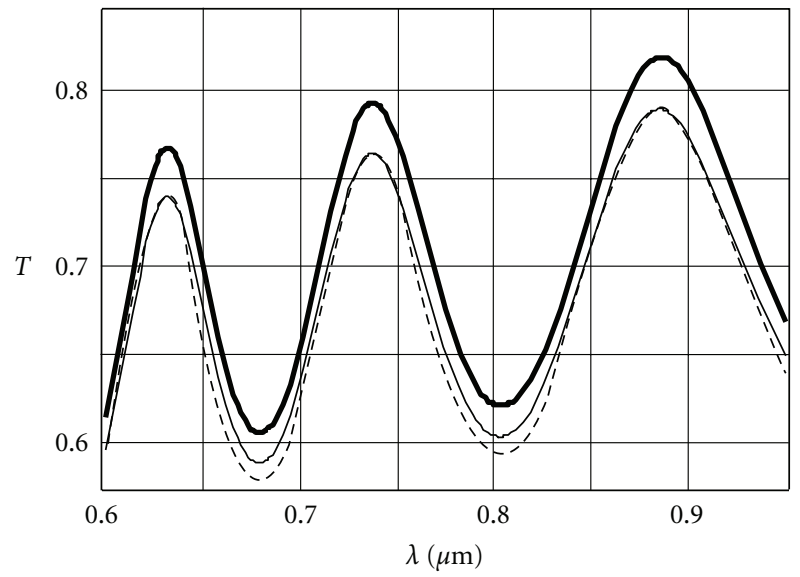

Figure 7: Transmittance spectrum of a thin dielectric film $\left(d_{1}=\right.$ $1 \mu \mathrm{m})$ with small optical losses $\left(n_{1}=2.21, k_{1}=0.01\right)$ on a transparent substrate $\left(n_{2}=1.472, k_{2}=0\right)$ computed for the $3 \mathrm{M}$ structure (solid line) and $4 \mathrm{M}$ structure (dashed line); a solid thin curve is calculated from (1) and (3) by using the same numerical values.

thin-line curve. For processing of this spectrum we have used the known iteration procedure $[20,21,27]$ which allows one to find values of $n_{1}\left(\lambda_{i}\right)$ and $k_{1}\left(\lambda_{i}\right)$ at points $\lambda_{i}(i=1,2, \ldots)$ of an experimental spectrum.

Unlike the above cited papers, we have applied our newly derived (93) where its coefficients for the $4 \mathrm{M}$ structure with the device averaging are given in the form of (49), (79), (80), (82), (84), and (95). The values of $n_{1}\left(\lambda_{i}\right)$ and $k_{1}\left(\lambda_{i}\right)$ obtained from the experimental spectrum $T(\lambda)$ (a thin curve in Figure 8) have been approximated in the form of (92) with coefficients found by the least-squares method, namely,

$$
n_{1}(\lambda)=\frac{0.003}{\lambda^{4.285}}+2.045, \quad k_{1}(\lambda)=\frac{0.00027}{\lambda^{2.381}}+0.00050
$$

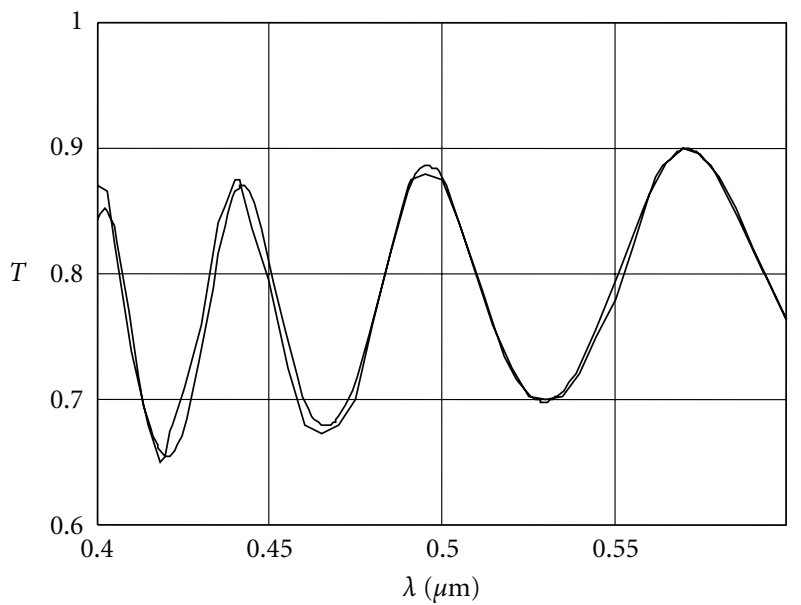

FIgURE 8: Experimental transmittance spectrum of an oxide film $\mathrm{Ta}_{2} \mathrm{O}_{5}$ on a quartz-glass substrate (thin line) and the spectrum $T(\lambda)$ calculated from (93) for the $4 \mathrm{M}$ structure (thick line) by using $d_{1}=$ $0.824 \mu \mathrm{m}, n_{1}(\lambda)$ and $k_{1}(\lambda)$ in the form of (97) which are obtained from the experimental spectrum.

where $\lambda$ is measured in $\mu m$. Thickness of the film $d_{1}$ has been proved to be equal to $0.824 \mu \mathrm{m}$ while the phase angle $\varphi_{+}$to be negligibly small because of small optical losses.

The spectrum $T(\lambda)$, calculated from our formulae by using $d_{1}=0.824 \mu \mathrm{m}$ and (97), is depicted by a thick curve in Figure 8. A close agreement between the experimental and calculated spectra $T(\lambda)$ justifies the practical applicability of our theoretical results to the processing of experimental optical spectra.

\section{Conclusion}

In the literature, there are no correct and consistent formulae relating to the reflectance and/or transmittance optical spectra for a thin film on a substrate of finite thickness, which are appropriate to the $4 \mathrm{M}$ structure model. A known model 
of the $3 \mathrm{M}$ structure developed previously in sufficient detail corresponds to the semiinfinite substrates and introduces an accuracy error into processing of measured optical spectra.

The paper has demonstrated a rigorous approach to development of a model for the $4 \mathrm{M}$-structure. The general expressions (43)-(59) for the spectra $R(\lambda)$ and $T(\lambda)$ are applicable to all kinds of the film-substrate structures including the particular case of semiinfinite substrates (see (63)-(69)). However, the general expressions derived for the $4 \mathrm{M}$ structure make no practical sense in the case of a sufficiently thick substrate because of appearing fast interference oscillations in the theoretical spectra. Any spectrophotometer with an operating slit of finite width eliminates such oscillations from the experimental spectra by means of their averaging because of nonmonochromatic irradiation (the so-called device averaging).

The crucial point of our theory is an introduction of the device averaging procedure into (43) in order to exclude the thick substrate oscillations nonrequired practically from theoretical spectra. This procedure has produced (60)-(61) for $R(\lambda)$ and $T(\lambda)$ in the simplest and succinct form to be convenient for processing of the experimental spectra. The newly derived equations have been analyzed, as applied to two special cases: (i) an arbitrary film on highly absorbing substrates and (ii) a slightly absorbing film on transparent substrates (see (78)-(84)). The results of such an analysis have displayed a close agreement between the obtained theoretical relations and experimental measurements.

Thus, the reflectance and transmittance spectra represented in the simplified (with the device averaging) form (60)-(61) are practically useful for determining the film thickness and optical constants from experimental spectra by applying the known techniques [20, 21, 23, 27]. Moreover, the more complex (without the device averaging) general form (43) may be applied to the 4M-structure (see Figure 1(b)) with a double film (of comparable values of $n_{1} d_{1}$ and $n_{2} d_{2}$ ) on semiinfinite substrates.

\section{Appendices}

\section{A. Derivation of Expressions for $\left|a_{4}\right|^{2}$ and $\left|c_{4}\right|^{2}$}

A.1. General Relations. Let us begin with employing (11), (13), (15), and (18) to explicitly write down parameters of the Fresnel reflection coefficient (19), that is,

$$
r_{m}=\bar{\rho}_{m} \mathrm{e}^{i \bar{\phi}_{m}} \equiv s_{m} \rho_{m} \mathrm{e}^{i s_{m} \phi_{m}},
$$

as applied to three interfaces $(m=1,2,3)$ which exist in the $4 \mathrm{M}$-structure depicted in Figure 1(b), then

(i) for interface 1 with $\bar{\rho}_{1}=s_{1} \rho_{1}$ and $\bar{\phi}_{1}=s_{1} \phi_{1}$

$$
\begin{gathered}
\bar{\rho}_{1}^{2}=\rho_{1}^{2}=\frac{\left|n_{0}-\tilde{n}_{1}\right|^{2}}{\left|n_{0}+\tilde{n}_{1}\right|^{2}} \equiv \frac{\mathcal{N}_{-1}}{\mathcal{N}_{+1}} \\
\tan \phi_{1}=\frac{2 n_{0} k_{1}}{\left.\left|n_{0}^{2}-\right| \tilde{n}_{1}\right|^{2} \mid},
\end{gathered}
$$

$$
\begin{gathered}
\mathcal{N}_{ \pm 1}=\left(n_{0}^{2}+\left|\tilde{n}_{1}\right|^{2}\right) \pm 2 n_{0} n_{1}, \\
s_{1}=\operatorname{sgn}\left\{n_{0}^{2}-\left|\tilde{n}_{1}\right|^{2}\right\},
\end{gathered}
$$

(ii) for interface 2 with $\bar{\rho}_{2}=s_{2} \rho_{2}$ and $\bar{\phi}_{2}=s_{2} \phi_{2}$

$$
\begin{gathered}
\bar{\rho}_{2}^{2}=\rho_{2}^{2}=\frac{\left|\tilde{n}_{1}-\tilde{n}_{2}\right|^{2}}{\left|\tilde{n}_{1}+\tilde{n}_{2}\right|^{2}} \equiv \frac{\mathcal{N}_{-2}}{\mathcal{N}_{+2}}, \\
\tan \phi_{2}=\frac{2\left(n_{1} k_{2}-n_{2} k_{1}\right)}{\left.|| \tilde{n}_{1}\right|^{2}-\left|\tilde{n}_{2}\right|^{2} \mid}, \\
\mathcal{N}_{ \pm 2}=\left(\left|\tilde{n}_{1}\right|^{2}+\left|\tilde{n}_{2}\right|^{2}\right) \pm 2\left(n_{1} n_{2}+k_{1} k_{2}\right), \\
s_{2}=\operatorname{sgn}\left\{\left|\tilde{n}_{1}\right|^{2}-\left|\tilde{n}_{2}\right|^{2}\right\},
\end{gathered}
$$

(iii) for interface 3 with $\bar{\rho}_{3}=s_{3} \rho_{3}$ and $\bar{\phi}_{3}=s_{3} \phi_{3}$

$$
\begin{gathered}
\bar{\rho}_{3}^{2}=\rho_{3}^{2}=\frac{\left|\tilde{n}_{2}-\tilde{n}_{3}\right|^{2}}{\left|\tilde{n}_{2}+\tilde{n}_{3}\right|^{2}} \equiv \frac{\mathcal{N}_{-3}}{\mathcal{N}_{+3}}, \\
\tan \phi_{3}=\frac{2\left(n_{2} k_{3}-n_{3} k_{2}\right)}{\left.|| \tilde{n}_{2}\right|^{2}-\left|\tilde{n}_{3}\right|^{2} \mid}, \\
\mathcal{N}_{ \pm 3}=\left(\left|\tilde{n}_{2}\right|^{2}+\left|\tilde{n}_{3}\right|^{2}\right) \pm 2\left(n_{2} n_{3}+k_{2} k_{3}\right), \\
s_{3}=\operatorname{sgn}\left\{\left|\tilde{n}_{2}\right|^{2}-\left|\tilde{n}_{3}\right|^{2}\right\} .
\end{gathered}
$$

For subsequent transformations it is necessary to employ the following quantities made up on the basis of (A.2)-(A.4), namely,

$$
\begin{aligned}
& \bar{\rho}_{1} \bar{\rho}_{2}=\frac{s_{1} s_{2}}{\mathcal{N}_{+1} \mathcal{N}_{+2}} S_{12} \quad \text { with } S_{12}=\sqrt{\mathcal{N}_{-1} \mathcal{N}_{+1} \mathcal{N}_{-2} \mathcal{N} \mathcal{N}_{+2}} \\
& \bar{\rho}_{1} \bar{\rho}_{3}=\frac{s_{1} s_{3}}{\mathcal{N}_{+1} \mathcal{N}_{+3}} S_{13} \quad \text { with } S_{13}=\sqrt{\mathcal{N}_{-1} \mathcal{N}_{+1} \mathcal{N}_{-3} \mathcal{N}_{+3}} \\
& \bar{\rho}_{2} \bar{\rho}_{3}=\frac{s_{2} s_{3}}{\mathcal{N}_{+2} \mathcal{N}_{+3}} S_{23} \quad \text { with } S_{23}=\sqrt{\mathcal{N}_{-2} \mathcal{N}_{+2} \mathcal{N}_{-3} \mathcal{N}_{+3}}
\end{aligned}
$$

It is convenient to rewrite (39) for $\left|a_{4}\right|^{2}$ and $\left|c_{4}\right|^{2}$ by using (33)-(34) and (40) in the following form:

$$
\begin{gathered}
\left|a_{4}\right|^{2}=\mathcal{A}_{\mathrm{abs}}+\mathcal{A}_{\mathrm{int}}+\bar{\rho}_{3} f_{a}\left(\beta_{2} d_{2}\right), \\
\left|c_{4}\right|^{2}=\mathcal{C}_{\mathrm{abs}}+\mathcal{C}_{\mathrm{int}}+\bar{\rho}_{3} f_{c}\left(\beta_{2} d_{2}\right),
\end{gathered}
$$

where the terms $\bar{\rho}_{3} f_{a}\left(\beta_{2} d_{2}\right)$ and $\bar{\rho}_{3} f_{c}\left(\beta_{2} d_{2}\right)$ have the previous form (41), while the absorption terms are

$$
\begin{aligned}
\mathcal{A}_{\mathrm{abs}}= & \left(\mathrm{e}^{2 \alpha_{1} d_{1}}+\bar{\rho}_{1}^{2} \bar{\rho}_{2}^{2} \mathrm{e}^{-2 \alpha_{1} d_{1}}\right) \mathrm{e}^{2 \alpha_{2} d_{2}} \\
& +\bar{\rho}_{3}^{2}\left(\bar{\rho}_{1}^{2} \mathrm{e}^{-2 \alpha_{1} d_{1}}+\bar{\rho}_{2}^{2} \mathrm{e}^{2 \alpha_{1} d_{1}}\right) \mathrm{e}^{-2 \alpha_{2} d_{2}}, \\
\mathcal{C}_{\mathrm{abs}}= & \left(\bar{\rho}_{1}^{2} \mathrm{e}^{2 \alpha_{1} d_{1}}+\bar{\rho}_{2}^{2} \mathrm{e}^{-2 \alpha_{1} d_{1}}\right) \mathrm{e}^{2 \alpha_{2} d_{2}} \\
& +\bar{\rho}_{3}^{2}\left(\mathrm{e}^{-2 \alpha_{1} d_{1}}+\bar{\rho}_{1}^{2} \bar{\rho}_{2}^{2} \mathrm{e}^{2 \alpha_{1} d_{1}}\right) \mathrm{e}^{-2 \alpha_{2} d_{2}},
\end{aligned}
$$


and the interference terms are

$$
\begin{aligned}
\mathcal{A}_{\text {int }}= & 2 \bar{\rho}_{1} \bar{\rho}_{2}\left\{\mathrm{e}^{2 \alpha_{2} d_{2}} \cos \left[\left(2 \beta_{1} d_{1}-\bar{\phi}_{1}\right)-\bar{\phi}_{2}\right]\right. \\
& \left.+\bar{\rho}_{3}^{2} \mathrm{e}^{-2 \alpha_{2} d_{2}} \cos \left[\left(2 \beta_{1} d_{1}-\bar{\phi}_{1}\right)+\bar{\phi}_{2}\right]\right\}, \\
\mathcal{C}_{\text {int }}= & 2 \bar{\rho}_{1} \bar{\rho}_{2}\left\{\mathrm{e}^{2 \alpha_{2} d_{2}} \cos \left[\left(2 \beta_{1} d_{1}+\bar{\phi}_{1}\right)-\bar{\phi}_{2}\right]\right. \\
& \left.+\bar{\rho}_{3}^{2} \mathrm{e}^{-2 \alpha_{2} d_{2}} \cos \left[\left(2 \beta_{1} d_{1}+\bar{\phi}_{1}\right)+\bar{\phi}_{2}\right]\right\} .
\end{aligned}
$$

We now pursue further transformations beginning with the interference terms (A.10).

A.2. Transformation of Interference Terms. Using (A.4) and (A.5), we can rewrite (A.10) in the form

$$
\begin{aligned}
& \mathcal{A}_{\text {int }}=\frac{4}{\mathcal{N}_{+1} \mathcal{N}_{+2} \mathcal{N}_{+3}} s_{1} s_{2} S_{12} f_{+}\left(\beta_{1} d_{1}\right), \\
& \mathcal{C}_{\text {int }}=\frac{4}{\mathcal{N}_{+1} \mathcal{N}_{+2} \mathcal{N}_{+3}} s_{1} s_{2} S_{12} f_{-}\left(\beta_{1} d_{1}\right),
\end{aligned}
$$

where we have defined the new quantities as functions of optical thickness $\beta_{1} d_{1}$ of layer 1

$$
\begin{aligned}
f_{ \pm}\left(\beta_{1} d_{1}\right)=\frac{1}{2}\{ & \mathcal{N}_{+3} \mathrm{e}^{2 \alpha_{2} d_{2}} \cos \left[\left(2 \beta_{1} d_{1} \mp \bar{\phi}_{1}\right)-\bar{\phi}_{2}\right] \\
& \left.+\mathcal{N}_{-3} \mathrm{e}^{-2 \alpha_{2} d_{2}} \cos \left[\left(2 \beta_{1} d_{1} \mp \bar{\phi}_{1}\right)+\bar{\phi}_{2}\right]\right\} .
\end{aligned}
$$

After some trigonometrical transformations, (A.12) take the form

$$
\begin{aligned}
f_{ \pm}\left(\beta_{1} d_{1}\right)= & \left(A \cos \bar{\phi}_{2}\right) \cos \left(2 \beta_{1} d_{1} \mp \bar{\phi}_{1}\right) \\
& +\left(B \sin \bar{\phi}_{2}\right) \sin \left(2 \beta_{1} d_{1} \mp \bar{\phi}_{1}\right) \\
\equiv & \sqrt{\left(A \cos \bar{\phi}_{2}\right)^{2}+\left(B \sin \bar{\phi}_{2}\right)^{2}} \cos \left(2 \beta_{1} d_{1}-\varphi_{ \pm}\right),
\end{aligned}
$$

where we have denoted

$$
\begin{aligned}
A \equiv & \frac{1}{2}\left(\mathcal{N}_{+3} \mathrm{e}^{2 \alpha_{2} d_{2}}+\mathcal{N}_{-3} \mathrm{e}^{-2 \alpha_{2} d_{2}}\right) \\
= & \left(\left|\tilde{n}_{2}\right|^{2}+\left|\tilde{n}_{3}\right|^{2}\right) \cosh 2 \alpha_{2} d_{2} \\
& +2\left(n_{2} n_{3}+k_{2} k_{3}\right) \sinh 2 \alpha_{2} d_{2}, \\
B \equiv & \frac{1}{2}\left(\mathcal{N}_{+3} \mathrm{e}^{2 \alpha_{2} d_{2}}-\mathcal{N}_{-3} \mathrm{e}^{-2 \alpha_{2} d_{2}}\right) \\
= & \left(\left|\tilde{n}_{2}\right|^{2}+\left|\tilde{n}_{3}\right|^{2}\right) \sinh 2 \alpha_{2} d_{2} \\
& +2\left(n_{2} n_{3}+k_{2} k_{3}\right) \cosh 2 \alpha_{2} d_{2} .
\end{aligned}
$$

The phase angles $\varphi_{ \pm}$appearing in (A.13) are defined as follows:

$$
\varphi_{ \pm}=\psi \pm \bar{\phi}_{1} \quad \text { with } \tan \psi=\frac{B}{A} \tan \bar{\phi}_{2}
$$

where $\tan \bar{\phi}_{2}$ follows from (A.3).

Substitution of (A.13) into (A.11) finally yields the desired expressions for the interference terms

$$
\begin{aligned}
& \mathcal{A}_{\text {int }}=\frac{4}{\mathcal{N}_{+1} \mathcal{N}_{+2} \mathcal{N}_{+3}} M \cos \left(2 \beta_{1} d_{1}-\varphi_{+}\right), \\
& \mathcal{C}_{\text {int }}=\frac{4}{\mathcal{N}_{+1} \mathcal{N}_{+2} \mathcal{N}_{+3}} M \cos \left(2 \beta_{1} d_{1}-\varphi_{-}\right),
\end{aligned}
$$

where

$$
M=s_{1} s_{2} S_{12} \sqrt{\left(A \cos \bar{\phi}_{2}\right)^{2}+\left(B \sin \bar{\phi}_{2}\right)^{2}}
$$

and from (A.2), (A.3), and (A.5), it follows that

$$
\begin{aligned}
S_{12} \equiv & \sqrt{\mathcal{N}_{-1} \mathcal{N}_{+1} \mathcal{N}_{-2} \mathcal{N}_{+2}} \\
=\{ & {\left[\left(n_{0}-n_{1}\right)^{2}+k_{1}^{2}\right]\left[\left(n_{0}+n_{1}\right)^{2}+k_{1}^{2}\right] } \\
& \times\left[\left(n_{1}-n_{2}\right)^{2}+\left(k_{1}-k_{2}\right)^{2}\right] \\
& \left.\times\left[\left(n_{1}+n_{2}\right)^{2}+\left(k_{1}+k_{2}\right)^{2}\right]\right\}^{1 / 2} .
\end{aligned}
$$

A.3. Transformation of Absorption Terms. Using relations (A.2)-(A.4) we can rewrite (A.9) in the form

$$
\begin{aligned}
& \mathcal{A}_{\mathrm{abs}}=\frac{4}{\mathcal{N}_{+1} \mathcal{N}_{+2} \mathcal{N}_{+3}} L_{+}, \\
& \mathcal{C}_{\mathrm{abs}}=\frac{4}{\mathcal{N}_{+1} \mathcal{N}_{+2} \mathcal{N}_{+3}} L_{-},
\end{aligned}
$$

where we have introduced the new quantities

$$
\begin{aligned}
L_{ \pm}= & \frac{1}{4}\left(\mathcal{N}_{ \pm 1} \mathcal{N}_{+2} \mathrm{e}^{2 \alpha_{1} d_{1}}+\mathcal{N}_{\mp 1} \mathcal{N}_{-2} \mathrm{e}^{-2 \alpha_{1} d_{1}}\right) \mathcal{N}_{+3} \mathrm{e}^{2 \alpha_{2} d_{2}} \\
& +\frac{1}{4}\left(\mathcal{N}_{\mp 1} \mathcal{N}_{+2} \mathrm{e}^{-2 \alpha_{1} d_{1}}+\mathcal{N}_{ \pm 1} \mathcal{N}_{-2} \mathrm{e}^{2 \alpha_{1} d_{1}}\right) \mathcal{N}_{-3} \mathrm{e}^{-2 \alpha_{2} d_{2}}
\end{aligned}
$$

After some transformations, (A.21) take the following form:

$$
\begin{aligned}
L_{ \pm}= & \frac{1}{2}\left(p_{ \pm} \mathcal{N}_{+3} \mathrm{e}^{2 \alpha_{2} d_{2}}+p_{\mp} \mathcal{N}_{-3} \mathrm{e}^{-2 \alpha_{2} d_{2}}\right) \cosh 2 \alpha_{1} d_{1} \\
& +\frac{1}{2}\left(q_{ \pm} \mathcal{N}_{+3} \mathrm{e}^{2 \alpha_{2} d_{2}}-q_{\mp} \mathcal{N}_{-3} \mathrm{e}^{-2 \alpha_{2} d_{2}}\right) \sinh 2 \alpha_{1} d_{1}
\end{aligned}
$$

where we have denoted

$$
\begin{aligned}
p_{ \pm} \equiv & \frac{1}{2}\left(\mathcal{N}_{ \pm 1} \mathcal{N}_{+2}+\mathcal{N}_{\mp 1} \mathcal{N}_{-2}\right) \\
= & \left(n_{0}^{2}+\left|\tilde{n}_{1}\right|^{2}\right)\left(\left|\tilde{n}_{1}\right|^{2}+\left|\tilde{n}_{2}\right|^{2}\right) \\
& \pm 4 n_{0} n_{1}\left(n_{1} n_{2}+k_{1} k_{2}\right), \\
q_{ \pm} \equiv & \frac{1}{2}\left(\mathcal{N}_{ \pm 1} \mathcal{N}_{+2}-\mathcal{N}_{\mp 1} \mathcal{N}_{-2}\right) \\
= & 2\left[\left(n_{0}^{2}+\left|\tilde{n}_{1}\right|^{2}\right)\left(n_{1} n_{2}+k_{1} k_{2}\right)\right. \\
& \left. \pm n_{0} n_{1}\left(\left|\tilde{n}_{1}\right|^{2}+\left|\tilde{n}_{2}\right|^{2}\right)\right]
\end{aligned}
$$


From (A.14) and (A.15), it follows that

$$
\mathcal{N}_{+3} \mathrm{e}^{2 \alpha_{2} d_{2}}=A+B, \quad \mathcal{N}_{-3} \mathrm{e}^{-2 \alpha_{2} d_{2}}=A-B .
$$

Insertion of (A.24) into (A.22) gives

$$
\begin{aligned}
L_{ \pm}= & \frac{1}{2}\left[\left(p_{ \pm}+p_{\mp}\right) A+\left(p_{ \pm}-p_{\mp}\right) B\right] \cosh 2 \alpha_{1} d_{1} \\
& +\frac{1}{2}\left[\left(q_{ \pm}-q_{\mp}\right) A+\left(q_{ \pm}+q_{\mp}\right) B\right] \sinh 2 \alpha_{1} d_{1} .
\end{aligned}
$$

The use of (A.23) for $p_{ \pm}$and $q_{ \pm}$reduces (A.25) to the final form

$$
L_{ \pm}=a_{ \pm} \cosh 2 \alpha_{1} d_{1}+b_{ \pm} \sinh 2 \alpha_{1} d_{1}
$$

where

$$
\begin{aligned}
a_{ \pm}= & \left(n_{0}^{2}+\left|\tilde{n}_{1}\right|^{2}\right)\left(\left|\tilde{n}_{1}\right|^{2}+\left|\tilde{n}_{2}\right|^{2}\right) A \\
& \pm 4 n_{0} n_{1}\left(n_{1} n_{2}+k_{1} k_{2}\right) B, \\
b_{ \pm}=2 & {\left[\left(n_{0}^{2}+\left|\tilde{n}_{1}\right|^{2}\right)\left(n_{1} n_{2}+k_{1} k_{2}\right) B\right.} \\
& \left. \pm n_{0} n_{1}\left(\left|\tilde{n}_{1}\right|^{2}+\left|\tilde{n}_{2}\right|^{2}\right) A\right]
\end{aligned}
$$

with the factors $A$ and $B$ introduced by (A.14) and (A.15).

Substitution of (A.26) into (A.20) yields the desired expressions for the absorption terms $\mathcal{A}_{\mathrm{abs}}$ and $\mathcal{C}_{\mathrm{abs}}$.

A.4. Transformation of the Functions $\bar{\rho}_{3} f_{a}\left(\beta_{2} d_{2}\right)$ and $\bar{\rho}_{3} f_{c}\left(\beta_{2} d_{2}\right)$. These functions are defined in (41). Using (A.2)-(A.3) and (A.6)-(A.7), we can rewrite these equations in the form

$$
\begin{aligned}
\bar{\rho}_{3} f_{a}\left(\beta_{2} d_{2}\right)= & \frac{4}{\mathcal{N}_{+1} \mathcal{N}_{+2} \mathcal{N}_{+3}} \\
& \times\left[s_{1} s_{3} S_{13} f_{+}\left(\beta_{2} d_{2}\right)+s_{2} s_{3} S_{23} g_{+}\left(\beta_{2} d_{2}\right)\right], \\
\bar{\rho}_{3} f_{c}\left(\beta_{2} d_{2}\right)= & \frac{4}{\mathcal{N}_{+1} \mathcal{N}_{+2} \mathcal{N}_{+3}} \\
& \times\left[s_{1} s_{3} S_{13} f_{-}\left(\beta_{2} d_{2}\right)+s_{2} s_{3} S_{23} g_{-}\left(\beta_{2} d_{2}\right)\right],
\end{aligned}
$$

where we have introduced the following new quantities as functions of optical thickness $\beta_{2} d_{2}$ of layer 2 (substrate)

$$
\begin{aligned}
& f_{ \pm}\left(\beta_{2} d_{2}\right)=\frac{1}{2}\{ \mathcal{N}_{+2} \cos \left[\left(2 \beta_{1} d_{1} \mp \bar{\phi}_{1}\right)+\left(2 \beta_{2} d_{2}-\bar{\phi}_{3}\right)\right] \\
&\left.+\mathcal{N}_{-2} \cos \left[\left(2 \beta_{1} d_{1} \mp \bar{\phi}_{1}\right)-\left(2 \beta_{2} d_{2}-\bar{\phi}_{3}\right)\right]\right\}, \\
& g_{ \pm}\left(\beta_{2} d_{2}\right)=\frac{1}{2}\left\{\mathcal{N}_{+1} \mathrm{e}^{ \pm 2 \alpha_{1} d_{1}} \cos \left[\left(2 \beta_{2} d_{2}-\bar{\phi}_{3}\right) \mp \bar{\phi}_{2}\right]\right. \\
&\left.+\mathcal{N}_{-1} \mathrm{e}^{\mp 2 \alpha_{1} d_{1}} \cos \left[\left(2 \beta_{2} d_{2}-\bar{\phi}_{3}\right) \pm \bar{\phi}_{2}\right]\right\} .
\end{aligned}
$$

After trigonometrical transformations (A.29), take the form

$$
\begin{aligned}
f_{ \pm}\left(\beta_{2} d_{2}\right)= & \left(\left|\tilde{n}_{1}\right|^{2}+\left|\tilde{n}_{2}\right|^{2}\right) \cos \left(2 \beta_{1} d_{1} \mp \bar{\phi}_{1}\right) \\
& \times \cos \left(2 \beta_{2} d_{2}-\bar{\phi}_{3}\right)-2\left(n_{1} n_{2}+k_{1} k_{2}\right) \\
& \times \sin \left(2 \beta_{1} d_{1} \mp \bar{\phi}_{1}\right) \sin \left(2 \beta_{2} d_{2}-\bar{\phi}_{3}\right) \\
g_{ \pm}\left(\beta_{2} d_{2}\right)= & \left(A_{ \pm} \cos \bar{\phi}_{2}\right) \cos \left(2 \beta_{2} d_{2}-\bar{\phi}_{3}\right) \\
& +\left(B_{ \pm} \sin \bar{\phi}_{2}\right) \sin \left(2 \beta_{2} d_{2}-\bar{\phi}_{3}\right) \\
\equiv & \sqrt{\left(A_{ \pm} \cos \bar{\phi}_{2}\right)^{2}+\left(B_{ \pm} \sin \bar{\phi}_{2}\right)^{2}} \\
& \times \cos \left[\left(2 \beta_{2} d_{2}-\bar{\phi}_{3}\right)-\psi_{ \pm}\right]
\end{aligned}
$$

where we have denoted

$$
\begin{aligned}
A_{ \pm} & \equiv \frac{1}{2}\left(\mathcal{N}_{+1} \mathrm{e}^{ \pm 2 \alpha_{1} d_{1}}+\mathcal{N}_{-1} \mathrm{e}^{\mp 2 \alpha_{1} d_{1}}\right) \\
& =\left(n_{0}^{2}+\left|\tilde{n}_{1}\right|^{2}\right) \cosh 2 \alpha_{1} d_{1} \pm 2 n_{0} n_{1} \sinh 2 \alpha_{1} d_{1} \\
B_{ \pm} & \equiv \pm \frac{1}{2}\left(\mathcal{N}_{+1} \mathrm{e}^{ \pm 2 \alpha_{1} d_{1}}-\mathcal{N}_{-1} \mathrm{e}^{\mp 2 \alpha_{1} d_{1}}\right) \\
& =\left(n_{0}^{2}+\left|\tilde{n}_{1}\right|^{2}\right) \sinh 2 \alpha_{1} d_{1} \pm 2 n_{0} n_{1} \cosh 2 \alpha_{1} d_{1} .
\end{aligned}
$$

The phase angles $\psi_{ \pm}$appearing in (A.31) are defined as

$$
\tan \psi_{ \pm}=\frac{B_{ \pm}}{A_{ \pm}} \tan \bar{\phi}_{2}
$$

where $\tan \bar{\phi}_{2}$ follows from (A.3).

Introducing the following denotation

$$
F_{ \pm}\left(\beta_{2} d_{2}\right)=s_{1} s_{3} S_{13} f_{ \pm}\left(\beta_{2} d_{2}\right)+s_{2} s_{3} S_{23} g_{ \pm}\left(\beta_{2} d_{2}\right),
$$

we can represent (A.28) in the form

$$
\begin{aligned}
& \bar{\rho}_{3} f_{a}\left(\beta_{2} d_{2}\right)=\frac{4}{\mathcal{N}_{+1} \mathcal{N}_{+2} \mathcal{N}_{+3}} F_{+}\left(\beta_{2} d_{2}\right), \\
& \bar{\rho}_{3} f_{c}\left(\beta_{2} d_{2}\right)=\frac{4}{\mathcal{N}_{+1} \mathcal{N}_{+2} \mathcal{N}_{+3}} F_{-}\left(\beta_{2} d_{2}\right) .
\end{aligned}
$$

Insertion of (A.30) and (A.31) in (A.34) convert it into the following:

$$
\begin{aligned}
F_{ \pm}\left(\beta_{2} d_{2}\right)= & N_{ \pm} \cos \left[\left(2 \beta_{2} d_{2}-\bar{\phi}_{3}\right)-\psi_{ \pm}\right] \\
& +P \cos \left(2 \beta_{1} d_{1} \mp \bar{\phi}_{1}\right) \cos \left(2 \beta_{2} d_{2}-\bar{\phi}_{3}\right) \\
& -Q \sin \left(2 \beta_{1} d_{1} \mp \bar{\phi}_{1}\right) \sin \left(2 \beta_{2} d_{2}-\bar{\phi}_{3}\right)
\end{aligned}
$$

where

$$
\begin{gathered}
N_{ \pm}=s_{2} s_{3} S_{23} \sqrt{\left(A_{ \pm} \cos \bar{\phi}_{2}\right)^{2}+\left(B_{ \pm} \sin \bar{\phi}_{2}\right)^{2}} \\
P=\left(\left|\tilde{n}_{1}\right|^{2}+\left|\tilde{n}_{2}\right|^{2}\right) s_{1} s_{3} S_{13}, \quad Q=2\left(n_{1} n_{2}+k_{1} k_{2}\right) s_{1} s_{3} S_{13},
\end{gathered}
$$


and from (A.2)-(A.4), (A.6), and (A.7), it follows that

$$
\begin{aligned}
S_{13} \equiv & \sqrt{\mathcal{N}_{-1} \mathcal{N}_{+1} \mathcal{N}_{-3} \mathcal{N}_{+3}} \\
=\{ & {\left[\left(n_{0}-n_{1}\right)^{2}+k_{0}^{2}\right]\left[\left(n_{0}+n_{1}\right)^{2}+k_{0}^{2}\right] } \\
& \times\left[\left(n_{2}-n_{3}\right)^{2}+\left(k_{2}-k_{3}\right)^{2}\right] \\
& \left.\times\left[\left(n_{2}+n_{3}\right)^{2}+\left(k_{2}+k_{3}\right)^{2}\right]\right\}^{1 / 2}, \\
S_{23} \equiv & \sqrt{\mathcal{N}_{-2} \mathcal{N}_{+2} \mathcal{N}_{-3} \mathcal{N}_{+3}} \\
= & \left\{\left[\left(n_{1}-n_{2}\right)^{2}+\left(k_{1}-k_{2}\right)^{2}\right]\left[\left(n_{1}+n_{2}\right)^{2}+\left(k_{1}+k_{2}\right)^{2}\right]\right. \\
& \times\left[\left(n_{2}-n_{3}\right)^{2}+\left(k_{2}-k_{3}\right)^{2}\right] \\
& \left.\times\left[\left(n_{2}+n_{3}\right)^{2}+\left(k_{2}+k_{3}\right)^{2}\right]\right\}^{1 / 2} .
\end{aligned}
$$

Substitution of the above (A.17), (A.20), and (A.35) into (A.8) finally yields the required expressions

$$
\begin{aligned}
& \left|a_{4}\right|^{2}=\frac{4}{\mathcal{N}_{+1} \mathcal{N}_{+2} \mathcal{N}_{+3}}\left[L_{+}+M \cos \left(2 \beta_{1} d_{1}-\varphi_{+}\right)+F_{+}\left(\beta_{2} d_{2}\right)\right], \\
& \left|c_{4}\right|^{2}=\frac{4}{\mathcal{N}_{+1} \mathcal{N}_{+2} \mathcal{N}_{+3}}\left[L_{-}+M \cos \left(2 \beta_{1} d_{1}-\varphi_{-}\right)+F_{-}\left(\beta_{2} d_{2}\right)\right],
\end{aligned}
$$

where the quantities $L_{ \pm}, M$ and $F_{ \pm}\left(\beta_{2} d_{2}\right)$ are, respectively, given by (A.26), (A.18), (A.36), and their accompanying constituents.

A knowledge of both $\left|a_{4}\right|^{2}$ and $\left|c_{4}\right|^{2}$ is sufficient to determine the reflectance $R$ from (38). But for determining the transmittance $T$ from the same formula, it is necessary to know not only $\left|a_{4}\right|^{2}$ but also the transmission coefficients product

$$
\left|t_{1}\right|^{2}\left|t_{2}\right|^{2}\left|t_{3}\right|^{2}=\frac{4}{\mathcal{N}_{+1} \mathcal{N}_{+2} \mathcal{N}_{+3}} 16 n_{0}^{2}\left|\tilde{n}_{1}\right|^{2}\left|\tilde{n}_{2}\right|^{2}
$$

which is obtained from (6) for coefficients $t_{m}, m=1,2,3$

\section{B. Mathematical Justification of the Device Averaging}

The general (43)-(59) have been derived for the case of monochromatic irradiation at wavelength $\lambda=$ const. But experimentally, one deals with nonmonochromatic conditions when $\left(\lambda_{0}-\Delta \lambda\right)<\lambda<\left(\lambda_{0}+\Delta \lambda\right)$, which is generated by the spectrophotometer with an operating slit of finite width $\sim \Delta \lambda \lll \lambda_{0}$. In such a case, the device indicates some average value. Our subsequent task is to prove that the average value of $F_{ \pm}\left(\beta_{2} d_{2}\right)$ given by (52)-(59) is negligibly small for the $4 \mathrm{M}$ structure with a thick substrate such that

$$
\eta \equiv \frac{\beta_{2} d_{2}}{\beta_{1} d_{1}}=\frac{n_{2} d_{2}}{n_{1} d_{1}} \gg 1 .
$$

Denoting $x=\beta_{1} d_{1} \equiv(2 \pi / \lambda) n_{1} d_{1}$, it is easy to see that $X_{1} \leq x \leq X_{2}$ where

$$
X_{1,2}=\beta_{0} n_{1} d_{1}\left(1 \mp \frac{\Delta \lambda}{\lambda_{0}}\right) \quad \text { with } \beta_{0}=\frac{2 \pi}{\lambda_{0}} .
$$

Now, the average value of $F_{ \pm}\left(\beta_{2} d_{2}\right)$ can be defined as

$$
\left\langle F_{ \pm}\left(\beta_{2} d_{2}\right)\right\rangle=\frac{1}{\Delta X} \int_{X_{1}}^{X_{2}} F_{ \pm}(x) d x
$$

where

$$
\Delta X \equiv X_{2}-X_{1}=2 \beta_{0} n_{1} d_{1} \frac{\Delta \lambda}{\lambda_{0}} \ll 1,
$$

and in accordance with (52) and (B.1)

$$
\begin{aligned}
F_{ \pm}(x)= & N_{ \pm} \cos \left[\left(2 \eta x-\bar{\phi}_{3}\right)-\psi_{ \pm}\right] \\
& +P \cos \left(2 x \mp \bar{\phi}_{1}\right) \cos \left(2 \eta x-\bar{\phi}_{3}\right) \\
& -Q \sin \left(2 x \mp \bar{\phi}_{1}\right) \sin \left(2 \eta x-\bar{\phi}_{3}\right) .
\end{aligned}
$$

Transformations of the trigonometric functions appearing in (B.5) give the following results:

$$
\begin{aligned}
& \cos \left[\left(2 \eta x-\bar{\phi}_{3}\right)-\psi_{ \pm}\right] \\
& =\cos \left(\psi_{ \pm}+\bar{\phi}_{3}\right) \cos 2 \eta x+\sin \left(\psi_{ \pm}+\bar{\phi}_{3}\right) \sin 2 \eta x, \\
& \cos \left(2 x \mp \bar{\phi}_{1}\right) \cos \left(2 \eta x-\bar{\phi}_{3}\right) \\
& =\frac{1}{2}\left\{\cos \left[2(\eta-1) x+\left( \pm \bar{\phi}_{1}-\bar{\phi}_{3}\right)\right]\right. \\
& \left.+\cos \left[2(\eta+1) x+\left(\mp \bar{\phi}_{1}-\bar{\phi}_{3}\right)\right]\right\} \\
& \approx \cos \left( \pm \bar{\phi}_{1}\right) \cos \left(2 \eta x-\bar{\phi}_{3}\right) \\
& =\left(\cos \bar{\phi}_{1} \cos \bar{\phi}_{3}\right) \cos 2 \eta x \\
& +\left(\cos \bar{\phi}_{1} \sin \bar{\phi}_{3}\right) \sin 2 \eta x \\
& \sin \left(2 x \mp \bar{\phi}_{1}\right) \sin \left(2 \eta x-\bar{\phi}_{3}\right) \\
& =\frac{1}{2}\left\{\cos \left[2(\eta-1) x+\left( \pm \bar{\phi}_{1}-\bar{\phi}_{3}\right)\right]\right. \\
& \left.-\cos \left[2(\eta+1) x+\left(\mp \bar{\phi}_{1}-\bar{\phi}_{3}\right)\right]\right\} \\
& \approx \sin \left(\mp \bar{\phi}_{1}\right) \sin \left(2 \eta x-\bar{\phi}_{3}\right) \\
& = \pm\left(\sin \bar{\phi}_{1} \sin \bar{\phi}_{3}\right) \cos 2 \eta x \\
& \mp\left(\sin \bar{\phi}_{1} \cos \bar{\phi}_{3}\right) \sin 2 \eta x \text {. }
\end{aligned}
$$

Approximate equality in (B.7) and (B.8) corresponds to the condition $\eta \gg 1$ that holds true for thick substrates (see (B.1)). 
Substitution of (B.6)-(B.8) into (B.5) gives

$$
F_{ \pm}(x)=C_{ \pm} \cos 2 \eta x+S_{ \pm} \sin 2 \eta x
$$

where (see (53)-(59) for $N_{ \pm}, P$ and $Q$ )

$$
\begin{aligned}
& C_{ \pm}=N_{ \pm} \cos \left(\psi_{ \pm}+\bar{\phi}_{3}\right)+P \cos \bar{\phi}_{1} \cos \bar{\phi}_{3} \mp Q \sin \bar{\phi}_{1} \sin \bar{\phi}_{3} \\
& S_{ \pm}=N_{ \pm} \sin \left(\psi_{ \pm}+\bar{\phi}_{3}\right)+P \cos \bar{\phi}_{1} \sin \bar{\phi}_{3} \pm Q \sin \bar{\phi}_{1} \cos \bar{\phi}_{3} .
\end{aligned}
$$

By integration of (B.9) in accordance with (B.3), we arrive at the desired result for the averaged value of $F_{ \pm}\left(\beta_{2} d_{2}\right)$ :

$$
\begin{aligned}
\left\langle F_{ \pm}\left(\beta_{2} d_{2}\right)\right\rangle= & \left(C_{ \pm} \cos 2 \beta_{0} n_{2} d_{2}+S_{ \pm} \sin 2 \beta_{0} n_{2} d_{2}\right) \\
& \times \frac{\sin \eta \Delta X}{\eta \Delta X} \longrightarrow 0 \quad \text { as } \eta \Delta X \gg 1 .
\end{aligned}
$$

The similar procedure of averaging applied to the terms $M \cos \left(2 \beta_{1} d_{1}-\varphi_{ \pm}\right)$entering into (43) gives the following result:

$$
\begin{aligned}
& \left\langle M \cos \left(2 \beta_{1} d_{1}-\varphi_{ \pm}\right)\right\rangle \\
& =M \cos \left(2 \beta_{0} n_{1} d_{1}-\varphi_{ \pm}\right) \\
& \quad \times \frac{\sin \Delta X}{\Delta X} \longrightarrow M \cos \left(\frac{4 \pi}{\lambda_{0}} n_{1} d_{1}-\varphi_{ \pm}\right) \quad \text { as } \Delta X \ll 1 .
\end{aligned}
$$

Therefore, from (B.11) and (B.12), it follows that for the $4 \mathrm{M}$ structures with a thick substrate the averaged contribution to $(43)$ from $F_{ \pm}\left(\beta_{2} d_{2}\right)$ is negligibly small and can be omitted but the terms $M \cos \left(2 \beta_{1} d_{1}-\varphi_{ \pm}\right)$should be taken at the center wavelength $\lambda_{0}$.

In conclusion, it should be emphasized that the above mathematical justification is only an approximate estimation based on the additive averaged contributions to (43) for the reflectance and transmittance optical spectra.

\section{Acknowledgment}

The authors wish to acknowledge the Russian Foundation for Basic Research for financial support (Grant no. 09-03-00777a).

\section{References}

[1] M. Born and E. Wolf, Principles of Optics, Pergamon, Oxford, UK, 4th edition, 1968.

[2] S. Tolansky, Muliple-Beam Interferometry of Surfaces and Films, Oxford University, London, UK, 1948.

[3] H. Mayer, Physik Dünne Schichten, Wissenschaftliche Verlagsgesellschaft, Stuttgart, Germany, 1950.

[4] S. Methfessel, Dünne Schichten, VEB Wilhelm Knapp, Halle, Germany, 1953.

[5] O. S. Heavens, Optical Properties of Thin Solid Films, Butterworths, London, UK, 1955.

[6] A. Vasicek, Optics of Thin Films, North Holland, Amsterdam, The Netherlands, 1960.
[7] D. Clarke and J. F. Grainger, Polarized Light and Optical Measurements, Pergamon, Oxford, UK, 1971.

[8] F. Abeles, "Investigations on the propagation of sinusoidal electromagnetic waves in stratified media: application to thin films," Annales de Physique, vol. 5, pp. 596-640, 1950.

[9] P. H. Berning, "Theory and calculations of optical thin films," in Physics of Thin Films, G. Hass, Ed., vol. 1, pp. 69-121, Academic Press, New York, NY, USA, 1963.

[10] O. S. Heavens, "Measurement of optical constants of thin films," in Physics of Thin Films, G. Hass and R. E. Thun, Eds., vol. 2, pp. 193-238, Academic Press, New York, NY, USA, 1964.

[11] H. E. Bennett and J. M. Bennett, "Measurement of optical constants of thin films," in Physics of Thin Films, G. Hass and R. E. Thun, Eds., vol. 4, pp. 1-96, Academic Press, New York, NY, USA, 1967.

[12] F. Abeles, "Optical properties of metallic films," in Physics of Thin Films, M. H. Francombe and R. W. Hoffman, Eds., vol. 6, pp. 151-204, Academic Press, New York, NY, USA, 1967.

[13] N. R. Rama Rao, R. Chandramani, and G. M. Rao, “Tantalum oxide films prepared by unbalanced reactive magnetron sputtering," Journal of Materials Science Letters, vol. 18, no. 23, pp. 1949-1951, 1999.

[14] K. M. A. Salam, H. Konishi, M. Mizuno, H. Fukuda, and S. Nomura, "Structural and electrical properties of crystalline $(1-x) \mathrm{Ta}_{2} \mathrm{O}_{5}-x \mathrm{Tio}_{2}$ thin films fabricated by metalorganic decomposition," Applied Surface Science, vol. 190, no. 1-4, pp. 88-95, 2002.

[15] R. A. Silva, M. Walls, B. Rondot, M. Da Cunha Belo, and R. Guidoin, "Electrochemical and microstructural studies of tantalum and its oxidefilms for biomedical applications in endovascular surgery," Journal of Materials Science, vol. 13, pp. 495-500, 2002.

[16] N. Negishi, K. Takeuchi, and T. Ibusuki, "Preparation of the TiO thin film photocatalyst by the dip-coating process," Journal of Sol-Gel Science and Technology, vol. 13, no. 1-3, pp. 691-694, 1999.

[17] T. Watanabe, S. Fukayama, M. Miyauchi, A. Fujishima, and K. Hashimoto, "Photocatalytic activity and photo-induced wettability conversion of $\mathrm{TiO}_{2}$ thin film prepared by sol-gel process on a soda-lime glass," Journal of Sol-Gel Science and Technology, vol. 19, pp. 71-76, 2000.

[18] Z. Huang, P. C. Maness, D. M. Blake, E. J. Wolfrum, S. L. Smolinski, and W. A. Jacoby, "Bactericidal mode of titanium dioxide photocatalysis," Journal of Photochemistry and Photobiology A, vol. 130, no. 2-3, pp. 163-170, 2000.

[19] T. Takahashi, H. Nakabayashi, and W. Mizuno, "Effects of plasma exposure on structural and optical properties of $\mathrm{TiO}_{2}$ films deposited by off-axis target sputtering," Journal of Vacuum Science and Technology A, vol. 20, no. 6, pp. 19161920, 2002.

[20] J. F. Hall and W. F. C. Ferguson, "Optical properties of cadmium sulfide and zinc sulfide from 0.6 micron to 14 micron," Journal of the Optical Society of America, vol. 45, pp. 714-718, 1955.

[21] S. P. Lyashenko and V. K. Miloslavskii, "A simple method for the determination of thickness and optical constants of semiconductor and dielectric layers," Optics and Spectroscopy, vol. 16, pp. 80-81, 1964.

[22] J. C. Burgiel, Y. S. Chen, and F. Vratny, "Refractive index of $\mathrm{ZnO}, \mathrm{ZnS}$ and several thin-film insulators," Journal of The Electrochemical Society, vol. 115, pp. 729-732, 1968.

[23] J. C. Manifacier, J. Gasiot, and J. P. Fillard, "A simple method for the determination of the optical constants $n, k$ and the 
thickness of a weakly absorbing thin film," Journal of Physics E, vol. 9, no. 11, article no. 032, pp. 1002-1004, 1976.

[24] R. Swanepoel, "Determination of the thickness and optical constants of amorphous silicon," Journal of Physics E, vol. 16, no. 12 , article no. 023, pp. 1214-1222, 1983.

[25] R. Swanepoel, "Determining refractive index and thickness of thin films from wavelength measurements only," Journal of the Optical Society of America A, vol. 2, pp. 1339-1343, 1985.

[26] H. Demiryont, J. R. Sites, and K. Geib, "Effects of oxygen content on the optical properties of tantalum oxide films deposited by ion-beam sputtering," Applied Optics, vol. 24, pp. 490-495, 1985.

[27] K. Ayadi and N. Haddaoui, "New approach to the determination of optical constants and thickness of thin dielectric transparent films," Journal of Materials Science: Materials in Electronics, vol. 11, no. 2, pp. 163-167, 2000.

[28] N. Martin, C. Rousselot, C. Savall, and F. Palmino, "Characterizations of titanium oxide films prepared by radio frequency magnetron sputtering," Thin Solid Films, vol. 287, no. 1-2, pp. 154-163, 1996.

[29] Y. Q. Hou, D. M. Zhuang, G. Zhang, M. Zhao, and M. S. $\mathrm{Wu}$, "Influence of annealing temperature on the properties of titanium oxide thin film," Applied Surface Science, vol. 218, no. 1-4, pp. 97-105, 2003.

[30] M. Laurikaitis, J. Dudonis, and D. Milčius, "Deposition of zirconium oxynitride films by reactive cathodic arc evaporation and investigation of physical properties," Thin Solid Films, vol. 516, no. 7, pp. 1549-1552, 2008.

[31] S. V. Jagadeesh Chandra, S. Uthanna, and G. Mohan Rao, "Effect of substrate temperature on the structural optical and electrical properties of dc magnetron sputtered tantalum oxide films," Applied Surface Science, vol. 254, pp. 1953-1960, 2008.

[32] S. G. Tomlin, "More formulae relating to optical reflection and transmission by thin films," Journal of Physics D, vol. 5, no. 4, article no. 328, pp. 847-851, 1972. 

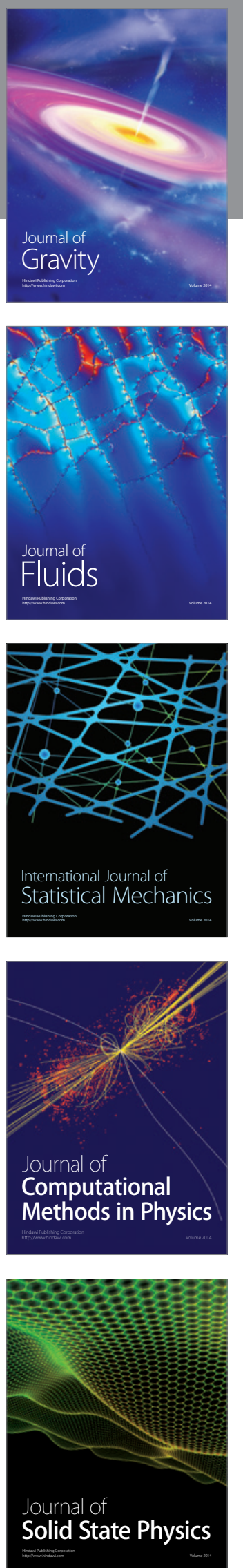

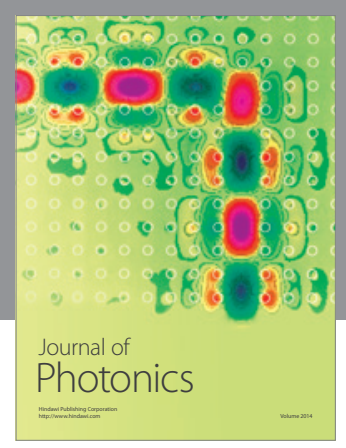

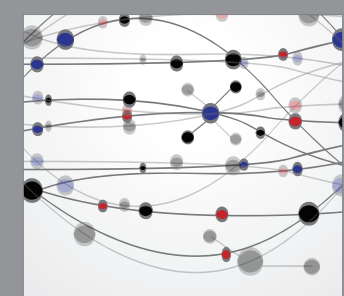

The Scientific World Journal
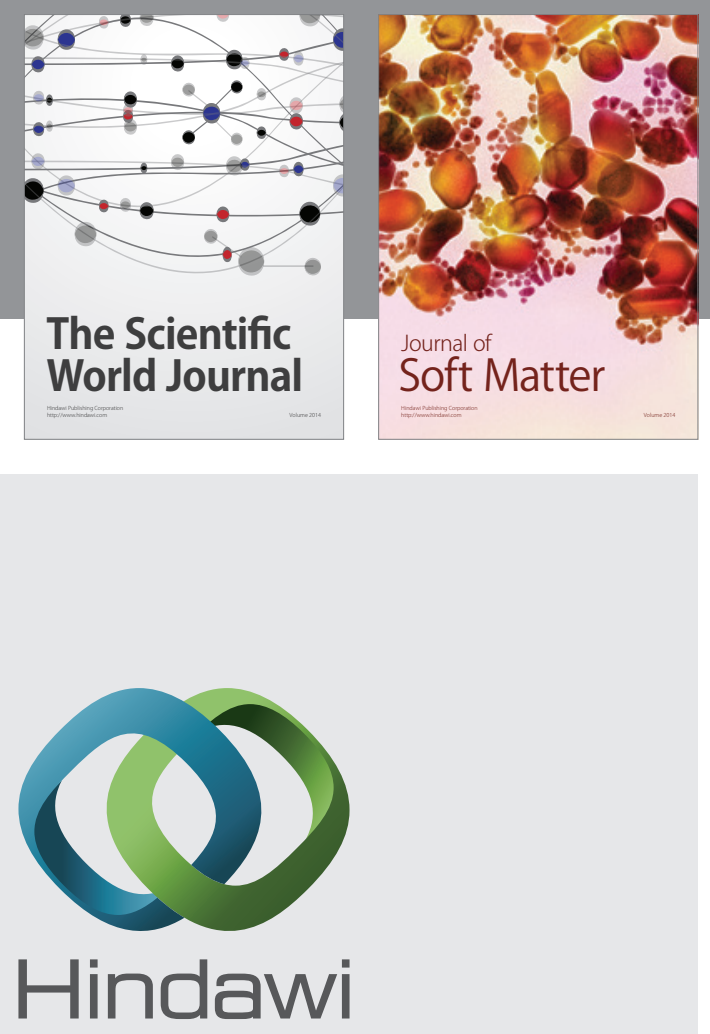

Submit your manuscripts at

http://www.hindawi.com
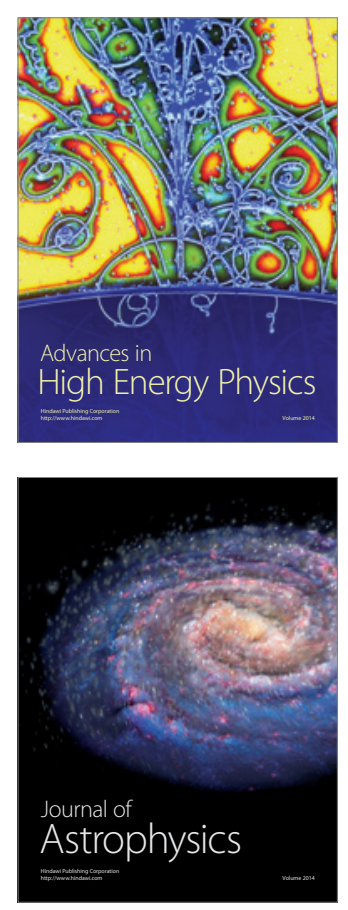
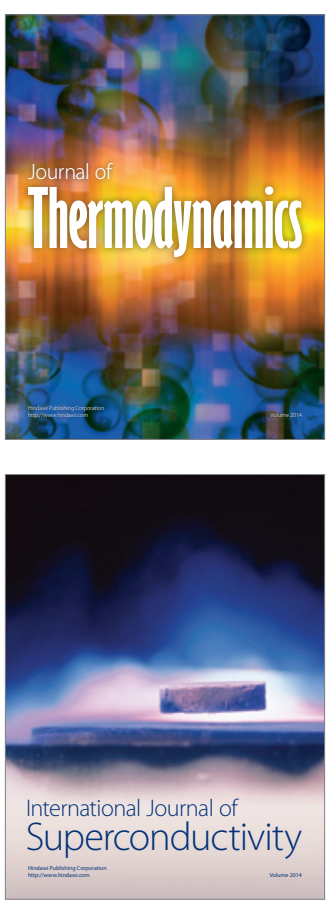
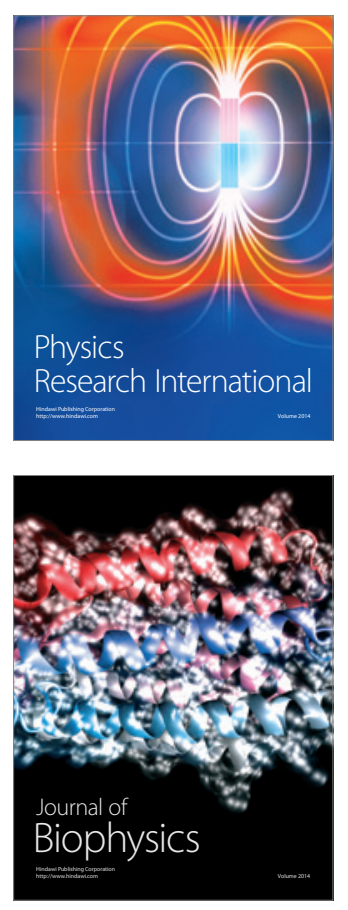
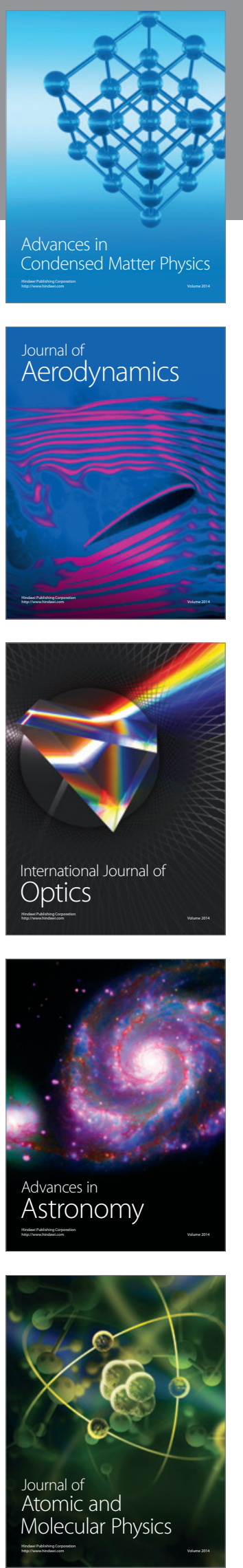